\title{
Multiple mechanism based constitutive modeling of gradient nanograined material
}

\author{
Jianfeng Zhao ${ }^{a}$, Xiaochong Lu ${ }^{a}$, Fuping Yuan ${ }^{\text {b,c }}$, Qianhua Kan ${ }^{\text {a }}$, Shaoxing Qu ${ }^{\text {d }}$, \\ Guozheng Kang ${ }^{\mathrm{a}}$, Xu Zhang ${ }^{\text {a," }}$ \\ a Applied Mechanics and Structure Safety Key Laboratory of Sichuan Province, School of Mechanics and Engineering, Southwest Jiaotong University, \\ Chengdu, 610031, China \\ b State Key Laboratory of Nonlinear Mechanics, Chinese Academy of Science, Institute of Mechanics, Beijing, China \\ ${ }^{\mathrm{c}}$ School of Engineering Science, University of Chinese Academy of Sciences, Beijing, China \\ ${ }^{\mathrm{d}}$ Key Laboratory of Soft Machines and Smart Devices of Zhejiang Province, Department of Engineering Mechanics, Zhejiang University, Hangzhou, \\ 310027, China
}

\section{A R T I C L E I N F O}

\section{Keywords:}

Gradient nano-grained materials

Geometrically necessary dislocations

Back stress

Deformation mechanism

Constitutive model

\begin{abstract}
A B S T R A C T
Gradient nano-grained (GNG) materials, inside which grain size increases gradually from nanoscale in the surface to micro-scale in the substrate, have shown synergetic strength and ductility. The extra strain hardening of GNG materials is considered to result from both geometrically necessary dislocations (GNDs) accommodating nonuniform plastic deformation and superior kinematic hardening characterized by back stress. However, few quantitative investigations were performed to evaluate the contribution of various strengthening mechanisms to the mechanical response of GNG materials. In this work, we develop a multiple-mechanism-based constitutive model, in which constitutive laws for GNDs and back stress at both grain level and sample level are established. Microstructure-based finite element simulation successfully predicts the uniaxial tensile behavior of a GNG interstitial-free (IF) steel sheet. The simulation results demonstrate that GNDs and back stress at sample level have little influence on the strengthening of the GNG IF-steel, while the back stress induced by pileup GNDs contributes about 35\% to the flow stress. The uniform elongation of the GNG sample is improved by the constraint of coarsegrained core on GNG layer. This work helps to understand the contributions of deformation mechanisms to the synergetic strength and ductility of GNG materials and to guide the microstructure design and optimization for improved strength-ductility combination.
\end{abstract}

\section{Introduction}

Mechanical properties of materials are dictated by their components, microstructures, and underlying deformation mechanisms. Great efforts have been made to adjust the components or microstructures to manufacture simultaneous strong and ductile materials (Cheng et al., 2018; Lu, 2014; Ma and Zhu, 2017; Ovid'ko et al., 2018). However, strength and ductility are always mutually exclusive. For instance, tuning the grain size of metals to nano-scale elevates their strength generally, but ductility decreases drastically since nano-grains retain less space to accommodate dislocations (Meyers et al., 2006). Gradient nano-grained (GNG) materials, inside which

\footnotetext{
* Corresponding author.

E-mail address: xzhang@swjtu.edu.cn (X. Zhang).
} 
grain size increases gradually from nano-scale in the surface to micro-scale in the substrate, have shown superior mechanical properties including high strength and hardness, considerable ductility and enhanced fatigue resistance (Cheng et al., 2018; Fang et al., 2011; Huang et al., 2015; Lin et al., 2018; Long et al., 2019; Shao et al., 2017; Wei et al., 2014; Wu et al., 2014a). For example, a GNG copper bar with GNG layer of volume fraction of $41.2 \%$ exhibits a yield strength of $~ 130 \mathrm{MPa}$, twice of its coarse-grained (CG) counterpart, meanwhile preserving a ductility of about 30\% (Fang et al., 2011). The yield strength of a GNG interstitial-free (IF) steel sheet is 1.6 times higher than its CG counterpart and reserves comparable uniform elongation (Wu et al., 2014a). Recently, the grain size of nickel was controlled by electrodeposition to prepare GNG architectures, which show enhanced strength-ductility synergy (Lin et al., 2018). The strength-ductility relations of homogeneously-grained and GNG metals discussed above are summarized in Fig. 1. Conspicuously, GNG metals show a linear relation between strength and ductility, instead of the nonlinear curve typical of homogeneously-grained ones. These results demonstrate that GNG materials exhibit a favorable strength-ductility combination superior to their homogeneously-grained counterparts.

The excellent strength-ductility synergy of GNG materials benefits from their unique microstructure. Due to the gradient distribution of grain size, heterogeneous deformation develops at two levels during deformation. At grain level, slip discontinuities arise near boundaries of adjacent grains, which are accommodated by GNDs, as schematically shown in Fig. 2(a). Although this also occurs in homogeneously-grained materials, larger deformation heterogeneity is introduced in GNG materials due to graded grain size. Therefore, in GNG materials studied here and other heterogeneous materials with mechanical incompatibilities between components inside, more GNDs are blocked in front of grain boundaries (GBS), giving rise to a stronger back stress strengthening than their CG counterparts (Hu et al., 2017; Liu et al., 2018; Wu and Zhu, 2017; Yang et al., 2016). At sample level, macroscopic plastic strain gradient develops in GNG materials due to the sequential yielding of different regions and strain localization occurring in the surface region (Shao et al., 2018; Wu et al., 2014a; Yuan et al., 2019). Experimental results demonstrated that strain localization occurs in the surface nano-grained region at an early deformation stage and then propagates along the tensile direction of a GNG IF-steel, while the deformation of CG core is more uniform (Yuan et al., 2019). The unique deformation features induce strain gradient along both the tensile direction and lateral direction. To accommodate these strain gradients, GNDs are generated (Wu et al., 2014a; Yuan et al., 2019), as shown in Fig. 2(b) and (c). Generally, strain gradient effect is considered when the density of GNDs is comparable to that of statistically stored dislocations (SSDs), such as metals at micro- or submicron-scale under nonuniform deformation (Fleck et al., 1994; Stolken and Evans, 1998). Therefore, in view of the deformation heterogeneities at both grain level and sample level, whether GNDs have an influence on the strain hardening of GNG materials and their quantitative contribution need to be clarified.

Physically-based constitutive modeling is an efficient way to investigate the effects of the involved multiple-deformationmechanisms on the mechanical response of materials. Considering the numerous grains in GNG metals, it is almost impossible to conduct a Voronoi-tessellation-based crystal plasticity finite element simulation reflecting the microstructure of a real GNG sample due to the computational burden (Wang et al., 2017; Zeng et al., 2015). Alternatively, some researchers adopted the rule of mixture (ROM) method (Li and Soh, 2012b; Li et al., 2017) to obtain the overall mechanical response of GNG materials. However, it has been proved that the ROM method underestimates the strength (Moering et al., 2016; Wu et al., 2014b; Yang et al., 2015), since the interaction between layers was neglected. The mutual constraint between different parts can be considered by a finite element simulation (Li and Soh, 2012a; Zhao et al., 2019). Dislocation-mechanism-based finite element modeling, enriched with grain-size dependent constitutive law for dislocation evolution, have been employed to predict the tensile mechanical behavior of GNG material, including yield strength, strain-hardening and ductility (Li and Soh, 2012a; Li et al., 2017; Lu et al., 2019b; Zhao et al., 2019). The constructed strength-ductility mappings, by manipulating the gradient distribution of grain size, also reveal the same inverse linear relationship observed experimentally, as shown in Fig. 1 (Lu et al., 2019b; Zhao et al., 2019). However, the GNDs induced by the heterogeneous deformation are always neglected. More importantly, no constitutive model has been established so far to predict high-level back stress in GNG materials.

In this paper, we aim to establish a multiple-mechanism-based constitutive model, which involves the effects of GNDs and back

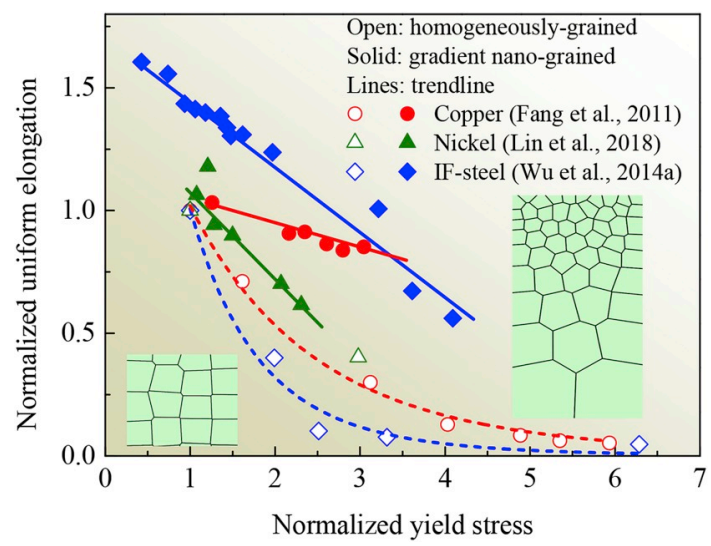

Fig. 1. Relationship between normalized uniform elongation and normalized yield stress of homogeneously-grained and gradient nano-grained copper (Fang, 2014), nickel (Lin et al., 2018) and interstitial-free steel (Wu et al., 2014a). 

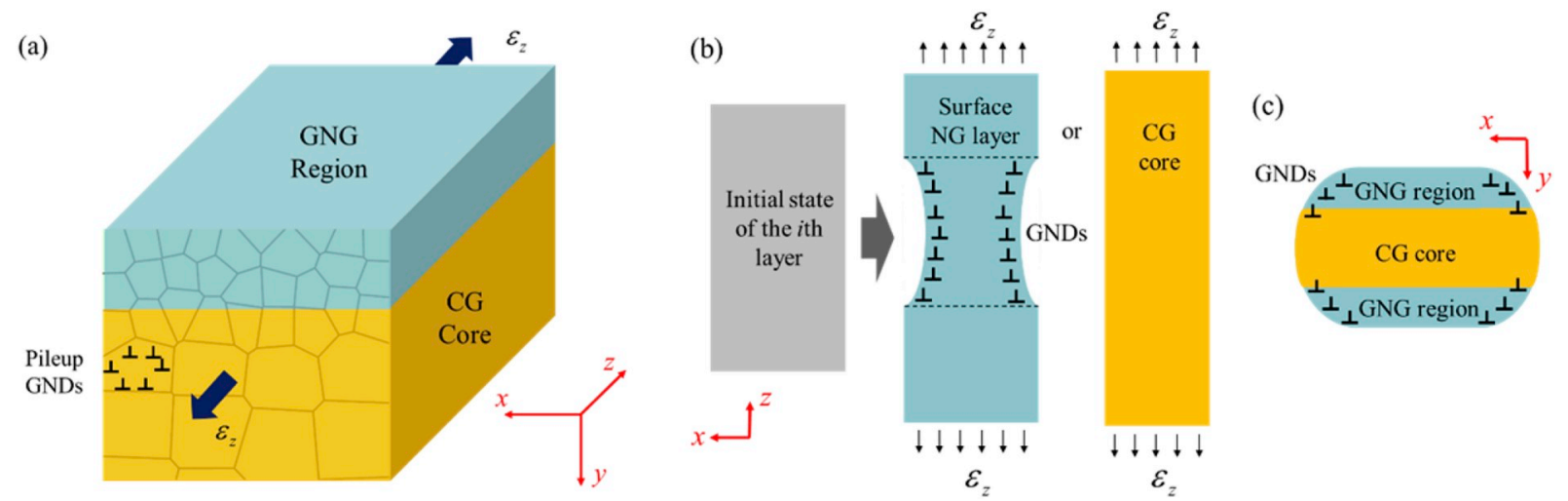

Fig. 2. (a) A GNG sheet under tensile deformation. At grain level, GNDs pile up at grain boundaries. (b) During tensile deformation, strain localization initiates in the surface nano-grained region at an early deformation stage, while the deformation of the CG core is more uniform. Sample-level GNDs are introduced along the tensile direction of the surface region (Yuan et al., 2019). (c) Shrinks of different parts in $x$-direction introduced GNDs along the depth direction.

stresses at both grain level and sample level, and to investigate the contributions of different deformation mechanisms to the strengthening of GNG materials. Following this goal, the paper is organized as follows. In section 2, the constitutive model for GNG materials is established. Section 3 describes the microstructure-based finite element model for a GNG IF-steel sheet under uniaxial tension. In Section 4, the simulated tensile response and its comparison with the experimental result are summarized, and the effects of multiple deformation mechanisms on the strength and ductility of GNG material are discussed. Finally, the study ends with some conclusions in section 5 .

\section{A mechanisms-based constitutive model for GNG materials}

In order to predict the relationship between different strengthening mechanisms and mechanical response of GNG materials, we develop a deformation-mechanism-based constitutive model. From a modeling point of view, the selection of internal variables is very important for constructing a constitutive model. For example, the original Taylor hardening model includes only one internal state variable, i.e., the overall dislocation density, as also adopted in this work. This one-variable model has been employed to model different kinds of metallic materials successfully (Estrin and Mecking, 1984; Kocks, 1976; Mecking and Kocks, 1981). Later, the one-variable model was extended to two-variable ones (Estrin et al., 1998; Mughrabi, 1983) by considering cell walls of high dislocation density and cell interiors with poor dislocations, or three-variable ones (Roters et al., 2000) by considering the mobile dislocations and immobile dislocations in both cell walls and the cell interiors. These kinds of multi-variable models are more elaborate since more detailed microstructure information are considered. However, more parameters are also brought in, accompanying the introduction of new internal state variables.

In this work, we intend to develop a constitutive model with a concise form since this model will be further considered to serve as a microstructure design tool for GNG materials. Therefore, only one internal variable, i.e., the dislocation density, is chosen as the microstructural state variable to make the model as concise as possible. Then, gain-size-dependent constitutive laws for both initial yielding and subsequent strain hardening are constructed to reflect the gradient structure in GNG materials. The effects of GNDs and back stress are also incorporated into this dislocation-based model.

\subsection{A viscoplastic framework for elastoplastic solids}

For an elastoplastic solid, the total strain rate can be decomposed into elastic and plastic parts,

$$
\dot{\varepsilon}_{i j} \quad \dot{\varepsilon}_{i j}^{\mathrm{e}} \quad \dot{\varepsilon}_{i j}^{\mathrm{p}} .
$$

Elastic Hooke's law gives the relationship between elastic strain rate and stress rate as

$$
\dot{\varepsilon}_{i j}^{\mathrm{e}} \quad \frac{1}{2 \mu} \dot{\sigma}_{i j}^{\prime} \quad \frac{\dot{\sigma}_{k k}}{9 K} \delta_{i j}
$$

where $\mu$ and $K$ are shear modulus and bulk modulus; $\dot{\sigma}_{i j}^{\prime} \quad \dot{\sigma}_{i j} \quad \dot{\sigma}_{k k} \delta_{i j} / 3$ is the deviatoric stress rate, $\dot{\sigma}_{k k}$ is the hydrostatic stress rate, and $\delta_{i j}$ is the Kronecker's symbol.

The plastic strain rate $\dot{\varepsilon}_{i j}^{\mathrm{p}}$ is related to the deviatoric stress according to the $J_{2}$-flow theory (Hill, 1950), 


$$
\dot{\varepsilon}_{i j}^{\mathrm{p}} \quad \frac{3 \dot{\varepsilon}^{\mathrm{p}}}{2 \sigma_{\mathrm{e}}} \sigma_{i j}^{\prime},
$$

where $\dot{\varepsilon}^{\mathrm{p}} \quad \sqrt{2} \dot{\varepsilon}_{i j}^{\mathrm{p}} \dot{\varepsilon}_{i j}^{\mathrm{p}} / 3$ is the effective plastic strain rate, $\sigma_{\mathrm{e}} \quad \sqrt{3} \sigma_{i j}^{\prime} \sigma_{i j}^{\prime} / 2$ denotes the von Mises effective stress. A power law viscoplastic formulation is used to correlate the effective plastic strain rate to the effective stress:

$$
\dot{\varepsilon}^{\mathrm{p}} \quad \dot{\varepsilon}_{0} \frac{\sigma_{\mathrm{e}}{ }^{m}}{\sigma_{\mathrm{f}}},
$$

where $\dot{\varepsilon}_{0}$ is the reference strain rate, the exponent $m$ describes rate-sensitivity and usually takes a large value (e.g., larger than 20 ), $\sigma_{\mathrm{f}}$ is the flow stress controlling plastic deformation, which is related to the microstructure and underlying deformation mechanisms of GNG materials. To eliminate strain rate and time dependence, $\dot{\varepsilon}_{0}$ is replaced by the effective strain rate $\dot{\varepsilon} \quad \sqrt{2} \dot{\varepsilon}_{i j} \dot{\varepsilon}_{i j} / 3$ (Kok et al., $2002 \mathrm{a}$, b), where $\dot{\varepsilon}_{i j} \quad \dot{\varepsilon}_{i j} \quad \dot{\varepsilon}_{k k} \delta_{i j} / 3$ denotes the deviatoric strain rate.

\subsection{Deformation mechanisms-based flow stress}

For GNG material, the flow stress which employs dislocation density as the internal variable controlling strain hardening is expressed in a general form of (Li and Soh, 2012b; Li et al., 2017; Zhu and Lu, 2012)

$$
\sigma_{\mathrm{f}} \quad \sigma_{\mathrm{Y}} \quad M \alpha \mu b \sqrt{ } \rho \quad \sigma_{\mathrm{b}}
$$

where $\sigma_{\mathrm{Y}}$ is initial yield stress, the Taylor hardening law (Taylor, 1934, 1938) gives the strain hardening resulting from dislocations, $M \alpha \mu b \sqrt{ } \rho$, where $\rho$ represents dislocation density, $M$ is the Taylor factor, $\alpha$ is a material constant and $b$ denotes the magnitude of the Burgers vector. In the established constitutive law for flow stress, both isotropic and kinematic hardenings are considered. Specifically, the first two terms in the right-hand side of Eq. (5) represent isotropic hardening, while kinematic hardening is reflected by back stress $\sigma_{\mathrm{b}}$.

Dislocations density is an important internal variable that characterizes the strain hardening of metallic material. In deformed metallic material, dislocations mainly accumulate by trapping each other in a random way, and the hardening is supposed to be caused only by SSDs. However, in GNG materials, GNDs are induced due to their heterogeneous deformation (Wu et al., 2014a; Yin et al., 2016; Yuan et al., 2019). As stated in the introduction, GNDs develop at two levels in GNG materials, i.e., sample level and grain level. Ashby (1970) showed a detailed schematic of the grain-level GNDs near GBs, as re-shown in the right half part of Fig. 3. In most cases, the grain-level GNDs are not considered in a constitutive model. However, the heterogeneous microstructure of GNG materials induces more pileup GNDs and produces strong back stress (Wu and Zhu, 2017; Yang et al., 2016), which suppresses the subsequent dislocation to further move toward GBs (Hirth and Lothe, 1982). Therefore, GND at the sample level and grain level are both considered.

On the other hand, the macroscopic deformation heterogeneities, as stated before, also require the accommodation of GNDs, i.e.,

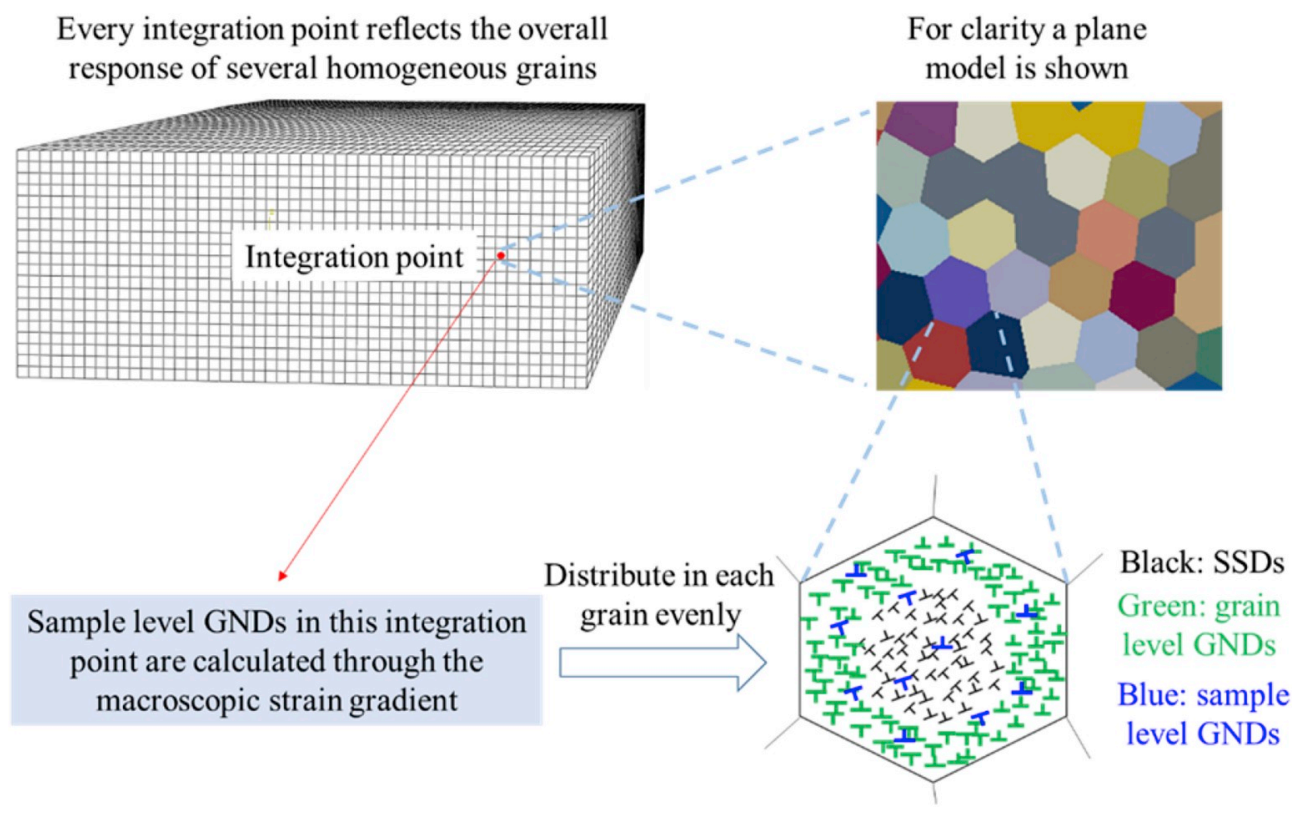

Fig. 3. Schematic of the total dislocation density considered in Taylor hardening law. 
the sample-level GNDs. The sample-level GNDs in each integration point can be calculated by the strain gradient. Since the integration point reflects the mechanical response of a cluster of homogeneous grains, these GNDs can be considered to be distributed evenly in the grain cluster, as shown in Fig. 3.

Ashby (1970) pointed that the total dislocation density can be calculated as $\rho \quad \rho_{\text {SSDs }} \quad \rho_{\mathrm{GNDs}}$. Considering that both grain-level GNDs and sample-level GNDs should be considered in GNG materials, the total dislocation density in each grain can be calculated as the summation of three parts, i.e.,

$$
\rho \quad \rho_{\mathrm{SSDs}} \quad \rho_{\mathrm{GNDs}}^{\mathrm{sam}} \quad \rho_{\mathrm{GNDs}}^{\mathrm{gra}}
$$

where $\rho_{\mathrm{SSDs}}, \rho_{\mathrm{GNDs}}^{\mathrm{sam}}, \rho_{\mathrm{GNDs}}^{\text {gra }}$ are the density of SSDs, GNDs at sample level and grain level, respectively.

The inhomogeneous distribution of the two-level GNDs will introduce the corresponding two levels of back stresses. Thus, the back stress in GNG material is comprised of,

$$
\sigma_{\mathrm{b}} \quad \sigma_{\mathrm{b}}^{\mathrm{sam}} \quad \sigma_{\mathrm{b}}^{\mathrm{gra}},
$$

where $\sigma_{\mathrm{b}}^{\mathrm{sam}}$ and $\sigma_{\mathrm{b}}^{\text {gra }}$ are back stresses at the sample level and at grain level, respectively.

Substitutions of Eq. (6) and Eq. (7) into Eq. (5), a comprehensive form of flow stress is expressed as

$$
\begin{array}{cccccc}
\sigma_{\mathrm{f}} & \sigma_{\mathrm{Y}} & M \alpha \mu b \sqrt{\rho_{\mathrm{SSDs}}} \quad \rho_{\mathrm{GNDs}}^{\mathrm{sam}} & \rho_{\mathrm{GNDs}}^{\mathrm{gra}} & \sigma_{\mathrm{b}}^{\mathrm{sam}} & \sigma_{\mathrm{b}}^{\text {gra }}
\end{array}
$$

It describes the individual contribution of different deformation mechanisms on the strain hardening behavior. However, the most distinctive feature of GNG materials, namely the information of gradient distribution of grain size, is absent. As is known, both the initial yielding and subsequent strain hardening behavior of materials depend on grain size (Armstrong, 1970; Meyers et al., 2006). Therefore, the dependences of yield stress, dislocation density, and back stress on grain size are established in the following subsections.

\subsubsection{Dependence of yield stress on grain size}

The pioneering works of Hall and Petch enable the description of yield stress regarding grain size, i.e., the Hall-Petch formula (Hall, 1951; Petch, 1953),

$$
\sigma_{\mathrm{Y}} \quad \sigma_{0} \frac{k_{\mathrm{HP}}}{\sqrt{ } d}
$$

where $\sigma_{0}$ is the lattice friction stress, $k_{\mathrm{HP}}$ is the Hall-Petch slope and $d$ denotes grain size, $k_{\mathrm{HP}} / \sqrt{ } d$ represents the strengthening effect from GBs. Note that for nanocrystalline material, the linear relation between $\sigma_{\mathrm{Y}}$ and $d^{1 / 2}$ breaks down (Meyers et al., 2006). A lot of experiments and modelings have reported that an inverse Hall-Petch relation exists when the grain size is reduced to tens of nanometers (Chokshi et al., 1989; Schiotz and Jacobsen, 2003). Therefore, for a GNG material with nano-grains in the surface, a modified Hall-Petch relation should be adopted (Zhao et al., 2019). In this work, the smallest grain size in the GNG IF-steel is about $100 \mathrm{~nm}$, so the classical Hall-Petch relation in Eq. (9) is still valid for describing the grain-size-dependent yield stress.

\subsubsection{Evolution of SSDs density with grain size}

The evolution of SSDs density for coarse/ultrafine-grained metals can be described according to the Kocks-Mecking-Estrin (KME) formula (Estrin, 1998; Kocks, 1976; Mecking and Kocks, 1981)

$$
\frac{\partial \rho_{\mathrm{SSDs}}}{\partial \varepsilon^{\mathrm{p}}} \quad M \frac{1}{b L} \quad k_{\mathrm{ann}} \rho_{\mathrm{SSDs}}
$$

The first term describes dislocation multiplication, which is associated with the mean free path of dislocations $L \quad 1 / \sqrt{ } \rho$. The second term denotes dislocation annihilation, which is assumed to be linear with dislocation density $\rho_{\text {SSDs }}$ with a proportional parameter $k_{\text {ann }}$. To describe the dependence of strain hardening on strain rate and temperature, $k_{\mathrm{ann}}$ is formulated as (Estrin, 1998)

$$
k_{\mathrm{ann}} \quad k_{\mathrm{ann}}^{0} \dot{\varepsilon}^{\mathrm{p}} \quad \dot{\varepsilon}_{\mathrm{ref}}{ }^{1 / n_{0}},
$$

where $k_{\text {ann }}^{0}$ is a material constant, $\dot{\varepsilon}_{\text {ref }}$ is the reference strain rate and $n_{0}$ is related to temperature. In the classical formula of the KME model, the multiplication and dynamic recovery process of dislocations didn't consider their dependence on grain size. Thus, in GNG material, it is desired to establish a modified evolution law for SSDs density, where the effect of GB on dislocation behaviors should be considered.

With the decrease of grain size, GBs become favorable to impede dislocation movement, so the influence of grain size on the mean free path of dislocations is considered, i.e.,

$$
\frac{1}{L} \frac{k_{\mathrm{mfp}}^{\mathrm{g}}}{d} \quad k_{\mathrm{mfp}}^{\mathrm{dis}} \sqrt{ } \rho_{\mathrm{SSDs}} \quad \rho_{\mathrm{GNDs}}
$$


where $\rho_{\mathrm{GNDs}} \quad \rho_{\mathrm{GNDs}}^{\mathrm{sam}} \quad \rho_{\mathrm{GNDs}}^{\mathrm{gra}}$ is the total GNDs density, $k_{\mathrm{mfp}}^{\mathrm{g}}$ is a geometrical parameter related to grain shape, $k_{\mathrm{mfp}}^{\text {dis }}$ is a proportionality factor. On the other hand, with the decrease of grain size, dislocation annihilations at GBs increase due to the enhanced absorption of dislocations by GBs. The annihilation rate of dislocation at GBs is described by $\rho_{\text {SSDs }} / t \dot{\varepsilon}^{\mathrm{p}}$ (Malygin, 2007; Valiev et al., 1994), where $t$ is the time required for dislocation dissolution at GBs. Different time is required for single dislocation and dislocation dipole to be dissolved. Specifically, for a single dislocation and a dislocation dipole, $t$ is in proportion to $d^{3}$ and $d^{2}$, respectively. It's known that GNDs are dislocations with polarities while SSDs are nonpolar dislocations overall. Considering that in a system including both positive and negative dislocations with densities of $\rho$ and $\rho$, if the total dislocation density has a polarity, then GNDs density can be calculated as $\rho_{\mathrm{GNDs}} \quad|\rho \quad \rho|$, and the left are SSDs with the same amount of positive and negative dislocations. Therefore, for the evolution of SSDs we consider dislocation dipole mechanism exclusively; the annihilation rate of dislocations at GBs is further given as $d_{\text {ref }} / d^{2} \rho_{\text {SSDs }}$ (Li and Soh, 2012b), where $d_{\text {ref }}$ represents the reference grain sizes below which the annihilations of dislocations intensify. Finally, a unified evolution of SSDs density for coarse/ultrafine-grained metals in GNG materials is given as

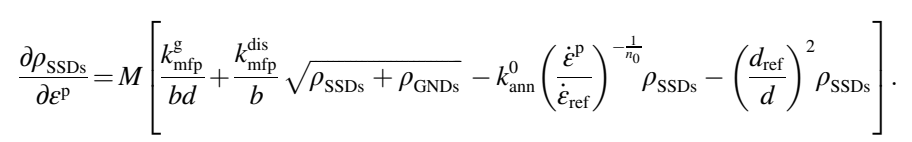

It should be noted that when grain size in the surface of a GNG material drops to nano-scale, GBs related deformation mechanisms become dominated (Khan and Liu, 2016; Van Swygenhoven, 2008). Therefore, for nanocrystal with a grain size of tens of nanometers, the dislocation density evolution law should be further established correspondingly (Malygin, 2007; Valiev et al., 1994). In this work, the smallest grain size in the studied GNG IF-steel is around $100 \mathrm{~nm}$; thus, the dislocation density evolution described by Eq. (13) is still valid.

\subsubsection{Evolutions of GNDs density and the resulting back stresses with grain size}

In this work, the GNG architecture is treated as a multilayer composite, which allows us to calculate the density of both grain-level GNDs and sample-level GNDs based on their generation mechanisms independently. The grain-level GNDs in a homogeneous polycrystalline will be calculated based on the pileup dislocations accommodating slip discontinuities between grains, and then the samplelevel GNDs can be considered as evenly distributed in the polycrystalline cluster in the integration point. The resulting back stresses are also established subsequently.

At the sample level, GNDs are required to accommodate deformation incompatibilities of different layers, which yield progressively or shrink differently in a GNG material. Nye (1953) and Ashby (1970) calculate the GNDs density by the effective plastic strain gradient $\eta^{\mathrm{p}}$

$$
\rho_{\mathrm{GNDs}}^{\mathrm{sam}} \bar{r} \frac{\eta^{\mathrm{p}}}{b}
$$

where $\bar{r}$ is the Nye factor. The effective plastic strain gradient $\eta^{\mathrm{p}}$ is defined as $\eta^{\mathrm{p}} \quad \sqrt{ } \eta_{i j k}^{\mathrm{p}} \eta_{i j k}^{\mathrm{p}} / 4$ (Fleck and Hutchinson, 1997), where $\eta_{i j k}^{\mathrm{p}} \quad \varepsilon_{i k, j}^{\mathrm{p}} \quad \varepsilon_{j k, i}^{\mathrm{p}} \quad \varepsilon_{i j, k}^{\mathrm{p}}$. In FEM implementation, macroscopic strain gradient (at sample level) is further realized through the gradient of shape function (Qu, 2004), e.g.,

$$
\varepsilon_{j k, i}^{\mathrm{p}} \quad \sum_{q 1}^{n} \frac{\partial N_{q}}{\partial x} \varepsilon_{j k}^{\mathrm{p}},
$$

where $N_{q}$ is the shape function and $n$ represents the number of Gaussian integration point in the adopted element. The index $i, j$, and $k$ correspond to $x, y$, and $z$ coordinate direction respectively.

For the back stress induced by GNDs, Evers et al. (2004a, b) have derived its shear components for edge and screw dislocations on a specific slip system. Later on, Bayley et al. (2006) proposed a back stress tensor induced by the spatial variation of GNDs density. Following Bayley et al. (2006) and assuming that the sample-level GNDs contain only edge type for simplification, which means the dislocation lines of GNDs are parallel to z-direction (loading direction) shown in Fig. 2 . Then the back stress tensor $\boldsymbol{\sigma}_{\mathrm{b}}^{\text {sam }}$ induced by GNDs at sample level can be calculated by

$$
\boldsymbol{\sigma}_{\mathrm{b}}^{\mathrm{sam}} \frac{\mu b R^{2}}{1 \nu}\left[\begin{array}{cccc}
\frac{3}{8} \frac{\partial \rho_{\mathrm{GNDs}}^{\mathrm{sam}}}{\partial y} & \frac{1}{8} \frac{\partial \rho_{\mathrm{GNDs}}^{\mathrm{sam}}}{\partial x} & 0 & \\
\frac{1}{8} \frac{\partial \rho_{\mathrm{GNDs}}^{\mathrm{sam}}}{\partial x} & \frac{1}{8} \frac{\partial \rho_{\mathrm{GNDs}}^{\mathrm{sam}}}{\partial y} & 0 & \\
0 & 0 & \frac{\nu}{2} \frac{\partial \rho_{\mathrm{GNDs}}^{\mathrm{sam}}}{\partial y}
\end{array}\right]
$$

where $\nu$ is the Poisson's ratio, $R$ is a cut-off radius of the circular domain within which GNDs contribute to back stress, and $x, y, z$ denote the lateral direction, depth direction and tensile direction of the GNG IF-steel sheet, as shown in Fig. 2(a). The effective back stress $\sigma_{\mathrm{b}}^{\text {sam }}$ can be calculated in a von Mises form as, 


$$
\sigma_{\mathrm{b}}^{\mathrm{sam}} \frac{\mu b R^{2}}{81 \nu} \sqrt{7} \quad 16 \nu^{2} \quad 16 \nu \quad \frac{\partial \rho_{\mathrm{GNDs}}^{\mathrm{sam}}}{\partial y}{ }^{2} \quad 3 \frac{\partial \rho_{\mathrm{GNDs}}^{\mathrm{sam}}}{\partial x}{ }^{2} .
$$

It will degrade into the expression given by Li et al. (2017) if the gradient of $\rho_{\mathrm{GNDs}}^{\mathrm{sam}}$ in lateral direction ( $x$-direction in Fig. 2), i.e., $\partial \rho_{\mathrm{GNDs}}^{\mathrm{sam}} / \partial x$, is neglected.

At grain level, GNDs pile up in front of GBs, their density can be calculated in an average way of

$$
\rho_{\mathrm{GNDs}}^{\mathrm{gra}} \frac{N}{d^{2}},
$$

where $d$ is the grain size, $N$ is the total number of piled up dislocations. Note that although other dislocation structures may be generated to accommodate strain heterogeneity between adjacent grains (Ashby, 1970), only pileup configuration is considered in this work for clarity and simplicity. The evolution of $N$ with plastic strain is proposed by Sinclair et al. (2006), in this work, it is modified to be,

$$
\frac{\partial N}{\partial \varepsilon^{\mathrm{p}}} \quad N_{\Delta} \quad 1 \frac{N}{N^{*}}
$$

where $N^{*}$ is the saturation number of dislocations that GBs can sustain, $N_{\Delta}$ the initial evolution rate of pileup dislocations. In larger grains, more space near GBs is provided for the formation of pileups. The saturation number of pileup dislocations $N^{*}$ should also have a positive correlation with grain size $d$. However, it is hard to acquire a precise correlation due to the lack of microscopic measurements and modelings. In this work, a linear relationship between $N^{*}$ and $d$ is assumed, i.e.,

$$
N^{*} \lambda d \quad N_{\text {extra }},
$$

where $\lambda$ is a proportional coefficient and $N_{\text {extra }}$ is a constant. Zhu et al.'s work (2012) also implies that grains with larger sizes possess larger $N_{\Delta}$. In this work, a linear relationship between $N_{\Delta}$ and $d$ is employed to deal with this relation, i.e.,

$$
N_{\Delta} \quad k_{\mathrm{N}} d \quad N_{\mathrm{A}} .
$$

It's noted that a special case of $N_{\mathrm{A}} 0$ has been adopted by Sinclair et al. (2006). Combinations of Eqs. (18)-(21) give a grain-size-dependent evolution law for pileup GNDs inside a grain as

$$
\frac{\partial \rho_{\mathrm{GNDs}}^{\mathrm{gra}}}{\partial \varepsilon^{\mathrm{p}}} \quad \frac{\partial \rho_{\mathrm{GNDs}}^{\mathrm{gra}}}{\partial N} \frac{\partial N}{\partial \varepsilon^{\mathrm{p}}} \frac{k_{\mathrm{N}} d N_{\mathrm{A}}}{d^{2}} 1 \frac{N}{\lambda d \quad N_{\text {extra }}} .
$$

The pileup GNDs inside a grain will generate back stress to suppress the subsequent dislocations to further move toward GBs. However, the distribution of GNDs in grains is very difficult to be captured. Assume the $N$ pileup edge dislocations distribute in a double-ended way that one half is on one side of GB and the other half is on the opposite side, then the induced back stress at grain level is calculated according to (Hirth and Lothe, 1982) as

$$
\sigma_{\mathrm{b}}^{\mathrm{gra}} \frac{M \mu b N}{\pi 1 \nu d}
$$

with the Taylor factor $M$ linking the macroscopic stress to the shear stress on slip systems.

This section established the grain-size-dependent evolution laws of yield stress, dislocation densities, and back stresses. In the following section, the model is implemented into the finite element software ABAQUS (2014) by using the user material subroutine to predict the overall mechanical response of a GNG IF-steel sheet under uniaxial tension.
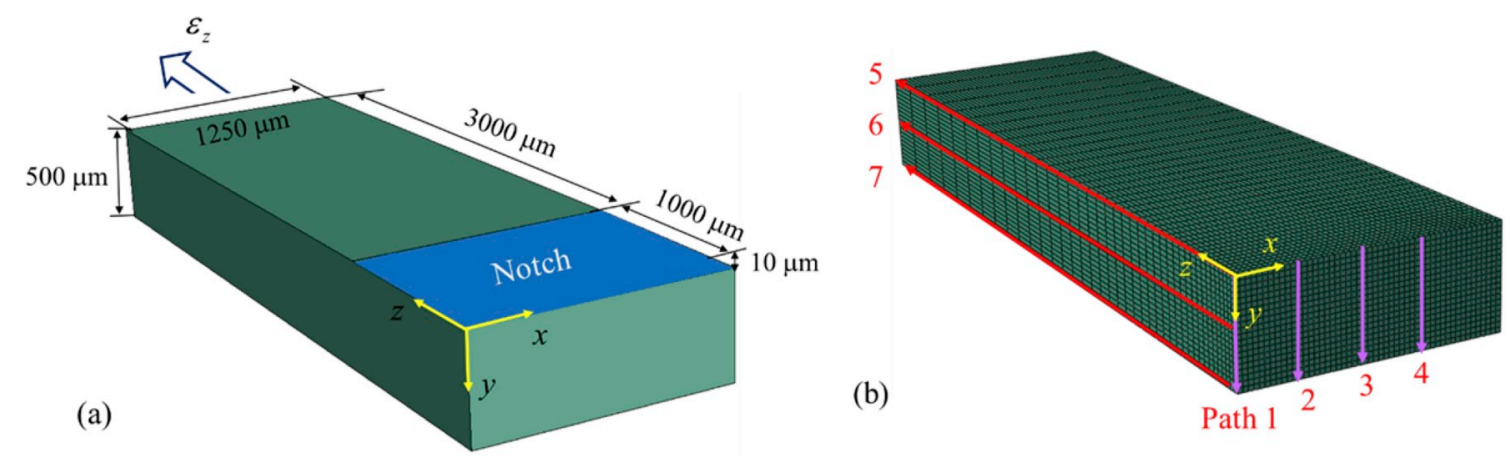

Fig. 4. (a) Geometry of the finite element model, a small notch is set in the surface region. (b) Finite element mesh of the model. 


\section{Microstructure-based FEM model for GNG IF-steel sheet under uniaxial tension}

\subsection{Geometric model and boundary conditions}

Wu et al. (2014a) fabricated a GNG IF-steel sheet by surface mechanical attrition treatment (SMAT), and tested its tensile mechanical property. To construct the finite element model reasonably, the model's geometry and boundary conditions should be consistent with the experimental one. The experimentally investigated GNG IF-steel sheet has a dimension of $2500 \mu \mathrm{m} \quad 1000$ $\mu \mathrm{m} \quad 8000 \mu \mathrm{m}\left(\begin{array}{lll}x & y & z\end{array}\right)(W u$ et al., 2014a). To simplify the simulation, it's reasonable that we used a 1/8 model (1250 $\mu \mathrm{m}$

$500 \mu \mathrm{m} \quad 4000 \mu \mathrm{m}$ ) with symmetric constraints applied, as shown in Fig. 4(a). Uniaxial tension with a strain rate of $5 \quad 10{ }^{4} / \mathrm{s}$ was applied along the $z$-direction.

SMAT inevitably introduces surface roughness into GNG IF-steel. To mimic the roughness and imperfection on the surface, an initial

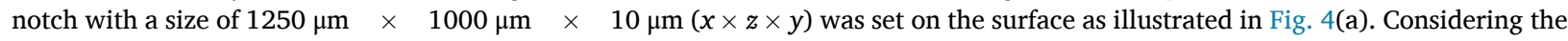
imperfect notch region in the nano-grained surface, we used a gradually refined mesh towards the notch with the smallest mesh size of $27 \mathrm{~nm}$. The overall finite element model was meshed with 8-node brick (C3D8) elements with 50 nodes in the $x$-direction, 20 nodes in $y$-direction and 40 nodes in z-direction to ensure the mesh independence of simulated results, as shown in Fig. 4(b).

\subsection{Microstructure-based multilayer composite}

The GNG architecture has been usually treated as a multilayer composite with each layer being an equivalent homogenous microstructure (Jin et al., 2018; Li et al., 2010; Li and Soh, 2012a, b; Li et al., 2017; Zhao et al., 2019), as shown in Fig. 5(a). To a certain degree, the accuracy of the modeling prediction depends on the number of discretized layers. Thus, a continuous distribution of grain size along depth is preferred. Based on the experimental results (Wu et al., 2014a) shown in Fig. 5(b), the grain size increases from $96 \mathrm{~nm}$ to $35 \mu \mathrm{m}$ along the depth direction, and the relation between the average grain size $d$ and the depth $h$ of the GNG IF-steel can be mathematically fitted as

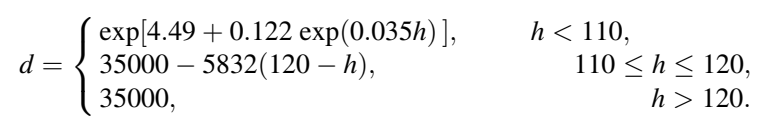

To reflect the real distribution of grain size in the investigated GNG IF-steel sheet, the above mathematical relations were adopted in the modeling.

\subsection{Constitutive parameters}

In the established constitutive model for GNG materials, most of the constitutive parameters are endowed with physical meanings, such as the shear modulus $G$, the magnitude of the Burgers vector $b$ and the Hall-Petch constant $k_{\mathrm{HP}}$, etc. The values of these parameters are almost constant and can be extracted from literature, as summarized in Table 1. Other constitutive parameters for GNG IF-steel are calibrated by simulating the tensile behavior of the homogeneously-grained IF-steel with grain sizes in the range from $100 \mathrm{~nm}$ to $35 \mu \mathrm{m}$, which are exactly the grain size in the topmost surface layer and bulk core respectively. The details of determining these parameters are given below.

Although there are eight parameters needed to be determined in this work, almost all of them can be obtained exclusively by the stress-strain curves of homogeneously-grained IF-steel with grain sizes of $96 \mathrm{~nm}, 650 \mathrm{~nm}$, and $35 \mu \mathrm{m}$. Firstly, we divide the eight

(a)

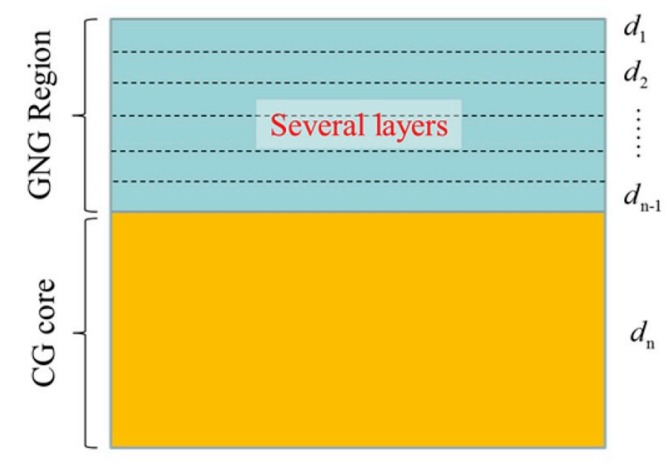

(b)

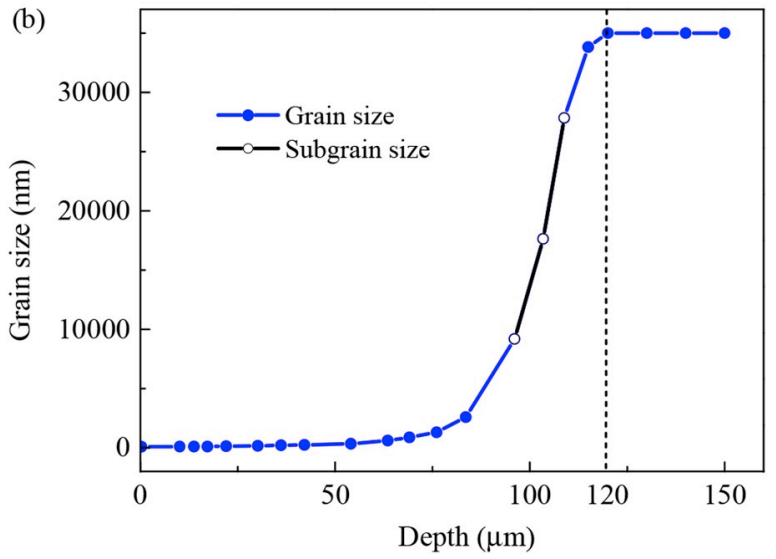

Fig. 5. (a) A multilayer structure of the GNG sample with different layers endowing different grain sizes. (b) Experimentally-measured grain size distribution function is used to obtain a continuous distribution of grain size along the depth direction (Wu et al., 2014 a). 
Table 1

Material parameters for the developed model in section 2.

\begin{tabular}{|c|c|c|c|}
\hline Parameter & Symbol & Value & Ref. \\
\hline Taylor factor & $M$ & 3.06 & \\
\hline Taylor constant & $\alpha$ & 0.3 & \\
\hline Shear modulus (GPa) & $G$ & 77 & Li et al. (2004) \\
\hline Poisson' ratio & $v$ & 0.28 & Li et al. (2017) \\
\hline Magnitude of Burgers vector (nm) & $b$ & 0.26 & Li et al. (2004) \\
\hline Lattice friction stress (MPa) & $\sigma_{0}$ & 65 & Wu et al. (2014a) \\
\hline Hall-Petch constant $1\left(\mathrm{MPa} \mu \mathrm{m}^{1 / 2}\right)$ & $k_{\mathrm{HP}}$ & 95 & Wu et al. (2014a) \\
\hline Rate sensitively exponent & $m$ & 20 & Huang et al. (2004) \\
\hline Geometric factor & $k_{\mathrm{mfp}}^{\mathrm{g}}$ & 0.06 & \\
\hline Proportionality factor & $k_{\mathrm{mfp}}^{\mathrm{dis}}$ & 0.008 & \\
\hline Dynamic recovery constant 1 & $k_{\mathrm{ann}}^{0}$ & 1.5 & \\
\hline Dynamic recovery constant 2 & $n_{0}$ & 21.25 & Li et al. (2017) \\
\hline Reference strain rate $\left(s^{1}\right)$ & $\dot{\varepsilon}_{\text {ref }}$ & 1 & Li et al. (2017) \\
\hline Reference grain size $(\mu \mathrm{m})$ & $d_{\text {ref }}$ & 2 & \\
\hline Nye-factor & $\bar{r}$ & 1.9 & Huang et al. (2004) \\
\hline Pileup dislocations constant $1\left(\mu \mathrm{m}{ }^{1}\right)$ & $k_{\mathrm{N}}$ & 46.15 & \\
\hline Pileup dislocations constant 2 & $N_{\mathrm{A}}$ & 307.7 & \\
\hline Pileup factor related to grain size $\left(\mu \mathrm{m}{ }^{1}\right)$ & $\lambda$ & 3.78 & \\
\hline Correction parameter of pileup dislocations & $N_{\text {extra }}$ & 0.62 & \\
\hline Cut-off radius of the GNDs domain $(\mu \mathrm{m})$ & $R$ & 3 & \\
\hline Initial dislocation density $\left(\mathrm{m}^{2}\right)$ & $\rho_{0}$ & $2 \quad 10^{11}$ & \\
\hline
\end{tabular}

parameters into two groups. The first group includes $k_{\mathrm{mfp}}^{\mathrm{g}}, k_{\mathrm{mfp}}^{\mathrm{dis}}, k_{\mathrm{ann}}^{0}$, and $d_{\text {ref }}$ as shown in Eq. (13), which describes the evolution of $\rho_{\text {SSDs }}$. The second group controlling the evolution of back stress includes $\lambda, N_{\text {extra }}, k_{\mathrm{N}}$ and $N_{\mathrm{A}}$, where $\lambda$ and $N_{\text {extra }}$ codetermine the saturation numbers of pileup dislocations; $k_{\mathrm{N}}$ and $N_{\mathrm{A}}$ describe the initial evolution rate of pileup dislocations.

Since the flow stresses shown in Fig. 6(a) include back stress, so the parameters related to the back stress in Table 1 are determined primarily. As indicated by Eq. (19), the back stress evolution is only dominated by $N$ and $N_{\Delta}$, so for IF-steel with specific grain size, e. g., $d \quad 35 \mu \mathrm{m}$, these two parameters can be exclusively determined by its back stress evolution curve. As shown in Eqs. (20) and (21), $N^{*} \quad \lambda d \quad N_{\text {extra }}, N_{\Delta} \quad k_{\mathrm{N}} d \quad N_{\mathrm{A}}$. Here, we determine $\lambda$ and $N_{\text {extra }}$ related to $N$ firstly. Two boundary conditions are required for determining $\lambda$ and $N_{\text {extra }}$. For the CG material with $d \quad 35 \mu \mathrm{m}$, as stated before, $N^{*}$ can be obtained by making a good fit to its back stress evolution curve (Wu et al., 2014a), which provides the first boundary condition. When the grain size drops to the nano-scale regime, the pileup mechanism breaks down, emission and absorption of dislocations at GBs are believed to dominate (Khan and Liu, 2016; Kumar et al., 2003; Van Swygenhoven, 2008). Therefore, for the IF-steel with a grain size of $100 \mathrm{~nm}$, we assume that only one dislocation can be piled up, which provides the second boundary condition, i.e., $d \quad 100 \mathrm{~nm}, N^{*} \quad 1$. With these two boundary conditions, $\lambda$ and $N_{\text {extra }}$ can be determined. $N_{\Delta}$, which controls the initial evolution rate of pileup dislocations, is equal to $N_{\Delta} \quad k_{N} d$ $N_{\mathrm{A}}$. When the grain size is very small, $N_{\Delta}$ is determined by $N_{\mathrm{A}}$, with the increase of grain size $N_{\Delta}$ becomes to be dominated by $k_{\mathrm{N}} d$. The stress-strain curve of IF-steel with a grain size of $100 \mathrm{~nm}$ shows that the stress saturates immediately after yielding, indicating the back stress also saturates at a small strain, which helps to determine $N_{\mathrm{A}}$. As $N_{\Delta}$ of the IF-steel with a grain size of $35 \mu \mathrm{m}$ has been obtained from the back stress evolution curve, then $k_{\mathrm{N}}$ can also be determined.
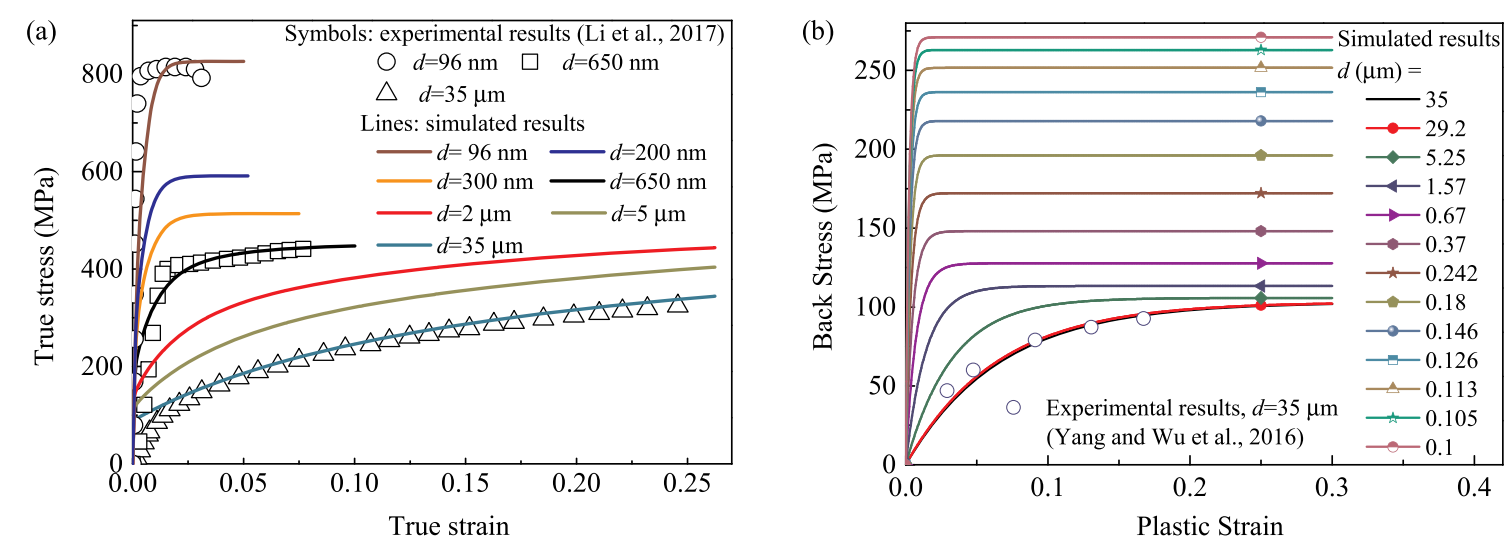

Fig. 6. The predicted curves of (a) true stress versus true strain and (b) back stress versus plastic strain for homogeneously-grained IF-steels with grain size changing from $100 \mathrm{~nm}$ to $35 \mu \mathrm{m}$, the experimental results are also shown for comparison (Wu et al., 2014a; Yang et al., 2016). 
As we have obtained the parameters related to the evolution of back stress, the back stress evolution curves for different grain sizes can be drawn. Then the stress-strain curves eliminating the influences of back stresses and grain-level GNDs are obtained. The strain hardening of these curves is dominated by $\rho_{\text {SSDs }}$. Although there are four parameters needed to be decided in the evolution law of $\rho_{\text {SSDs }}$, all of them can be determined exclusively since they are responsible for the description of the dislocation density evolution of different grain sizes clearly. For example, when the grain size is large enough, the influence of the first and fourth terms on the evolution of SSDs' density can be neglected. So the evolution of $\rho_{\mathrm{SSDs}}$ is merely controlled by $k_{\mathrm{mfp}}^{\mathrm{dis}}$ and $k_{\mathrm{ann}}^{0}$. Correspondingly, when the grain size is small enough, the influence of the second and third terms can be neglected, and the evolution of $\rho_{\text {SSDs }}$ is mainly controlled by $k_{\mathrm{mfp}}^{\mathrm{g}}$ and $d_{\mathrm{ref}}$. Note that the two sets of parameters are exclusive since for determining a nonlinear curve, we need two unique parameters. In this work, $k_{\mathrm{mfp}}^{\mathrm{dis}}$ and $k_{\mathrm{ann}}^{0}$ are determined by simulating the stress-strain curve of CG copper with a grain size of $35 \mu \mathrm{m}$ in Fig. $6(\mathrm{a}) ; k_{\mathrm{mfp}}^{\mathrm{g}}$ and $d_{\text {ref }}$ are obtained by simulating the copper with a grain size of $96 \mathrm{~nm}$. All the four parameters are adjusted slightly for making good fits to the three stress-strain curves of $35 \mu \mathrm{m}, 650 \mathrm{~nm}$, and $96 \mathrm{~nm}$ simultaneously in Fig. 6(a).

Once these parameters are determined, they are employed to model the GNG IF-steel with grain size gradually changing from $100 \mathrm{~nm}$ to $35 \mu \mathrm{m}$ from the surface to the core.

Fig. 6(a) indicates that the predicted results match the experimental data well for both the initial yielding and subsequent strain hardening. The yield strength increases from about $60 \mathrm{MPa}$ to $530 \mathrm{MPa}$ with the decrease of grain size from $35 \mu \mathrm{m}$ to $100 \mathrm{~nm}$, confirming the Hall-Petch relation. The strain hardening behavior becomes insignificant with the decrease of grain size since smaller grains have less space for dislocation accumulation. It is noted that the experimental data show that the elastic modulus seems to depend on the grain size. However, previous experimental and theoretical works (Kim and Bush, 1999; Sharma and Ganti, 2003) demonstrated that the elastic modulus decreases with the decrease of grain size, however, only in the nano-scale regime. So the deviation of elastic modulus may be attributed to the fluctuation during the preparation of IF steels. In this work, the elastic modulus we adopted coincides with that of the GNG IF-steel and thus is not tuned artificially to match the experiment for homogeneously-grained IF steel with different grain sizes. The predicted back stress evolution in Fig. 6(b) fits the experimental one well for IF-steel with a grain size of $35 \mu \mathrm{m}$. It is found that the evolution of back stress with strain is grain-size-dependent. When grain size is larger than $0.242 \mu \mathrm{m}$, it follows a nonlinear way. But for nanocrystal, it saturates immediately after the initiation of plastic deformation. This finding agrees with the experimental results, where the back stress of a CG copper increases with strain until a level of 20\% (Mahato et al., 2016), while the back stress saturates at a small strain of about $1 \%$ when nanoscale twins are induced to impede the dislocation movement (Wang et al., 2018). The modeling predicts that the saturation value of back stress increases from $100 \mathrm{MPa}$ to $270 \mathrm{MPa}$ with grain size decreases from $35 \mu \mathrm{m}$ to $100 \mathrm{~nm}$. The inverse dependence of $\sigma_{\mathrm{b}}^{\text {gra }}$ on grain size is consistent with the findings of experiments and discrete dislocation dynamics modeling, i.e., back stress introduced by pileup dislocations increases with the decrease of grain size (Espinosa et al., 2006; Lu et al., 2019a; Mahato et al., 2016; Massart and Pardoen, 2010).

The constitutive model with the calibrated constitutive parameters has successfully predicted the tensile response of homogeneously-grained IF-steel with grain size changing from $100 \mathrm{~nm}$ to several tens of microns. With such success, it will be further applied to simulate the tensile response of the GNG IF-steel sheet, and the relation between the underlying deformation mechanisms and macroscopic responses will be systematically investigated.

\section{Macroscopic mechanical behavior and strengthening mechanisms of GNG materials}

\subsection{Stress and strain response of the GNG IF-steel}

The uniaxial tensile response of GNG IF-steel under uniaxial tension is predicted using the developed model in Section 2 and the
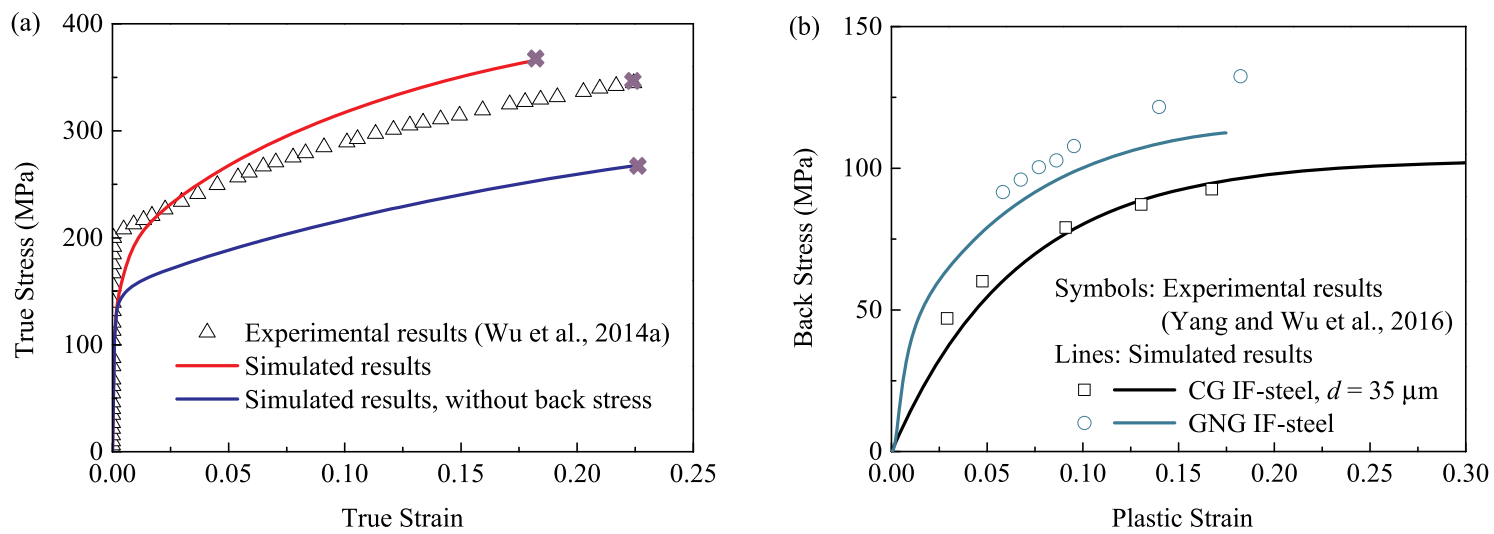

Fig. 7. (a) True stress versus true strain and (b) evolution of back stress predicted by the mechanism-based constitutive model for the GNG materials, the corresponding experimental results of the GNG IF-steel under tensile loading are also shown for comparison (Wu et al., 2014a; Yang et al., 2016). 
microstructure-based finite element model in Section 3. The same set of constitutive parameters, as used for homogeneously-grained IF-steel, are also used here for GNG IF-steel. Fig. 7(a) shows both the modeling predicted and experimentally measured true stressstrain curves of the GNG IF-steel under tensile loading. It turns out that the modeling predication is comparable to the experimental test. The predicted ultimate tensile strength of the GNG IF-steel is as high as $330 \mathrm{MPa}$, which is $\sim 20 \mathrm{MPa}$ higher than the experimental one; and the predicted uniform elongation is $\sim 18 \%$ (indicated by the cross, and the strain at this point is calculated by the relationship $\partial \sigma / \partial \varepsilon \quad \sigma$ ), which is smaller than the experimental one of $\sim 22 \%$ (Wu et al., 2014a). To systematically understand the deformation process of GNG materials, it is necessary to study the stress, strain and displacement field in the deformation stages following initial linear elastic deformation, i.e., elastic-plastic transition stage, plastic flow stage, plastic instability stage.

\subsubsection{Elastic-plastic transition stage}

Fig. 8 shows the distributions of stress/strain and displacement at a true tensile strain of $\varepsilon_{z} \quad 0.00287$. At this moment, the GNG layer deforms elastically while the CG region has already yielded, as manifested by the dash area in Fig. 8(a). Fig. 8(b) shows the absolute value of the lateral strain $\left|\varepsilon_{x}\right|$. It increases from the surface to the core, giving rise to a macroscopic strain gradient along the depth direction. The reason lies in that the plastically deformed CG core manifests a Poisson's ratio of 0.5 under the condition of plastic incompressibility, while the elastically deformed GNG layer retains a Poisson's ratio of 0.28 . So under tensile loading along the $z$ direction, the CG core shrinks more than the GNG layer in the lateral $(x)$ direction. The lateral displacement increases from about $1.73 \mu \mathrm{m}$ to $1.85 \mu \mathrm{m}$ from the surface to the core, which is quantitatively shown in Fig. 8(c). The incompatible deformation between the GNG layer and CG core leads to a conversion of the stress state from uniaxial to biaxial. As demonstrated by Fig. 8(d), the stress $\sigma_{x}$, which is supposed to be zero in homogeneously-grained material, is negative in the GNG region and positive in the CG core. Multiaxial stresses help to activate more slip systems, thus promotes dislocation accumulation to strengthen the GNG material (Wu et al., 2014a).

\subsubsection{Plastic deformation stage}

With the increase of tensile strain, the GNG layer also enters into plastic deformation regime. Due to the low strain hardening ability of top surface layers and the small notch in the surface region, strain localization occurs almost right after yielding in this region. The lateral strain $\varepsilon_{x}$ along the depth direction shown in Fig. 9(a) indicates that, contrary to what shown in Fig. 8(b), GNG layer shrinks more

(a)
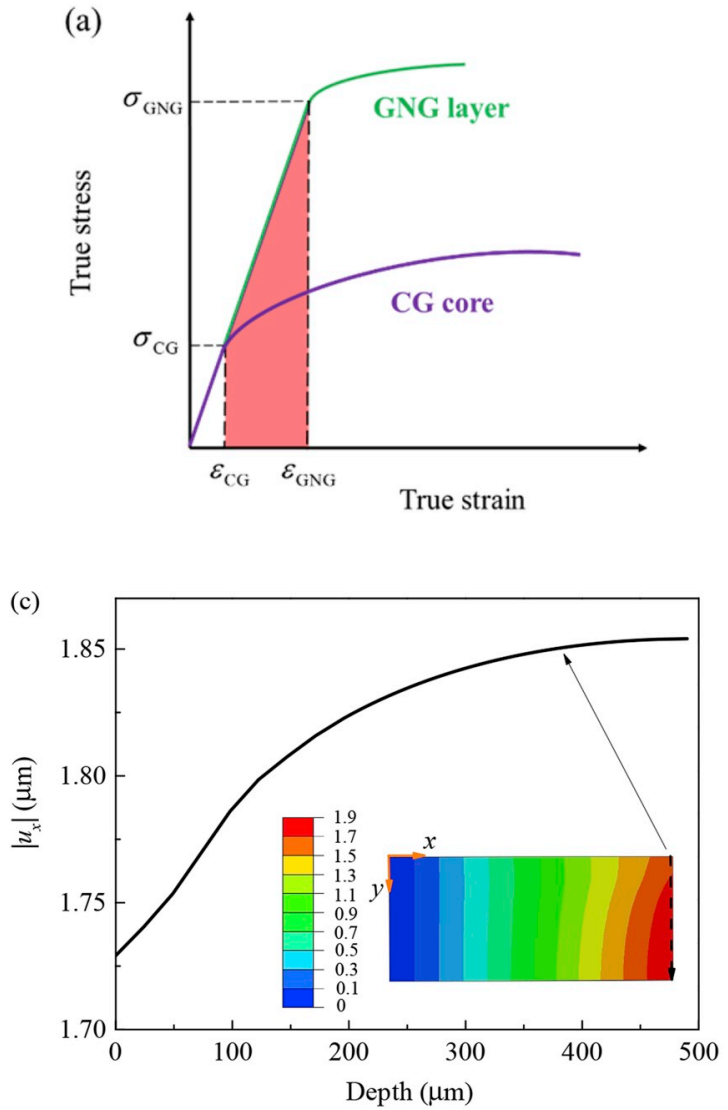
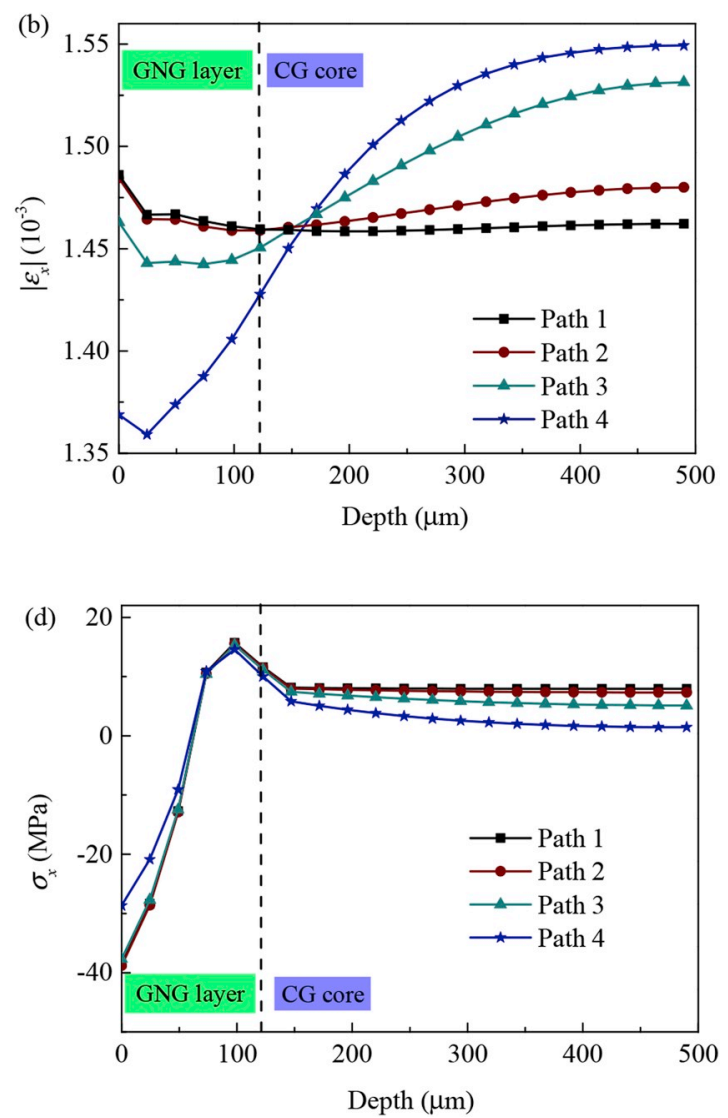

Fig. 8. (a) Schematic curves of true stress versus true strain of CG core and GNG layer, respectively. (b) Distribution of lateral strain $\varepsilon_{x}$ along the depth direction, where the four paths are marked in Fig. 4(b). (c) The contour of lateral displacement $u_{x}$ and its distribution along the depth direction. (d) Distribution of lateral stress $\sigma_{x}$ along path 1 to path 4 . 
significant than CG core at a true tensile strain of 0.05. Fig. 9(b) further gives the lateral displacement difference at different true strains. At a true strain of 0.00287 , the CG core shrinks about $0.12 \mu \mathrm{m}$ larger than the GNG layer since the plastic deformation occurs in the core region firstly. With the increase of tensile strain, strain localization occurs in the GNG layer. This region shrinks more significantly than the CG core, e.g., a maximum displacement difference of $0.77 \mu \mathrm{m}$ is predicted at a true strain of 0.05 . The elevated lateral displacement difference induces a raised strain gradient, which should be accommodated by GNDS.

\subsubsection{Plastic instability stage}

With the further increase of strain, the sample will lose stability. Experiments (Wu et al., 2014a; Yuan et al., 2019) demonstrate that although strain localization initiates in the surface region at an early deformation stage, the whole sample can sustain further deformation to a finial uniform elongation of about 0.22, as shown in Fig. 7(a). In the following, we reveal the underlying mechanisms for the superior ductility of GNG materials by analyzing the plastic instability occurring in the surface region and the whole sample. Fig. 10(a) shows the axial strain $\varepsilon_{z}$ along path 5, path 6, and path 7 as marked in Fig. 4(b) at different tensile strains. With the increase of tensile strain from 0.01 to 0.15 , the difference of $\varepsilon_{z}$ between uniform region and imperfect region along path 5 increases from 0.004 to 0.06 , indicating the evolution of nonuniform deformation in the surface region. Due to the mutual constraint between the GNG layer and CG core, the CG core also deforms nonuniformly. But from GNG surface to CG core, the degree of strain localization is reduced. For example, at a true strain of 0.15 , the strain difference between the strain localization region and the uniform region is 0.06 in the surface (path 5), while this value reduced to 0.046 in CG core (path 7).

Fig. 10(b) gives the evolutions of maximum and minimum axial strains $\varepsilon_{z \text {, max }}$ and $\varepsilon_{z \text {, min }}$ along path 5 with respect to the applied strain $\varepsilon_{\mathrm{z}}$. When $\varepsilon_{\mathrm{z}} \quad 0.007$ (marked by the blue spot), the evolution pathway of $\varepsilon_{\mathrm{z}, \max }$ starts to deviate from that of $\varepsilon_{z, \text { min. }}$ Such deviation indicates the beginning of strain localization in the surface. However, the strain localization in the surface region does not develop arbitrarily to result in the collapse of the whole GNG sample immediately. Both $\varepsilon_{z, \text { max }}$ and $\varepsilon_{z}$, min increase with the increase of the applied strain in an approximately linear way until $\varepsilon_{\mathrm{z}} \quad 0.18$, indicating the strain localization is effectively suppressed due to the constraint effect of CG core. This finding coincides with the result of molecular dynamics simulations (Lin et al., 2018), where the strain localization in the surface nano-grained region is inhibited by the mutual constraint between the GNG region and CG core. After $\varepsilon_{\mathrm{z}} \quad 0.18, \varepsilon_{\mathrm{z}, \max }$ increases faster while $\varepsilon_{z}$, min stops increasing with the further increase of $\varepsilon_{\mathrm{z}}$, and necking behavior initiates in the imperfect region where the notch is set.

\subsection{Distribution and evolution of deformation state variables}

The excellent mechanical responses of GNG materials benefit from their unique microstructures and deformation mechanisms. In order to obtain an in-depth and comprehensive understanding of the underlying mechanisms for the superior strain hardening, strength and ductility of the GNG IF-steel, deformation state variables such as SSDs, GNDs, and back stress, at different deformation stages are analyzed in detail.

\subsubsection{SSDs}

Fig. 11 shows the distribution and evolution of SSDs. When the tensile strain is lower than 0.03 , the density of SSDs in the GNG layer is larger than that in the CG core. It is because of the density of SSDs for small grains increases at a faster rate than large grains at smaller strains, coinciding with the stress-strain curves, as shown in Fig. 6(a). With the increase of tensile strain, the density of SSDs in the surface layer saturates while that in coarse grains continue to accumulate and exceed that in the surface layer. Furthermore, it is found that $\rho_{\text {SSDs }}$ increases from the GNG layer to CG core with a peak recorded at the border demarcating the GNG layer and CG core. For example, at the true strain of 0.18 , from the surface to the core $\rho_{\text {SSDs }}$ increases firstly from $6.4710^{12} / \mathrm{m}^{2}$ to $1.0210^{14} / \mathrm{m}^{2}$ and then decreases to $510^{13} / \mathrm{m}^{2}$. Experimentally, a peak of hardness increment is also detected there (Wu et al., 2014a). Recall the stress
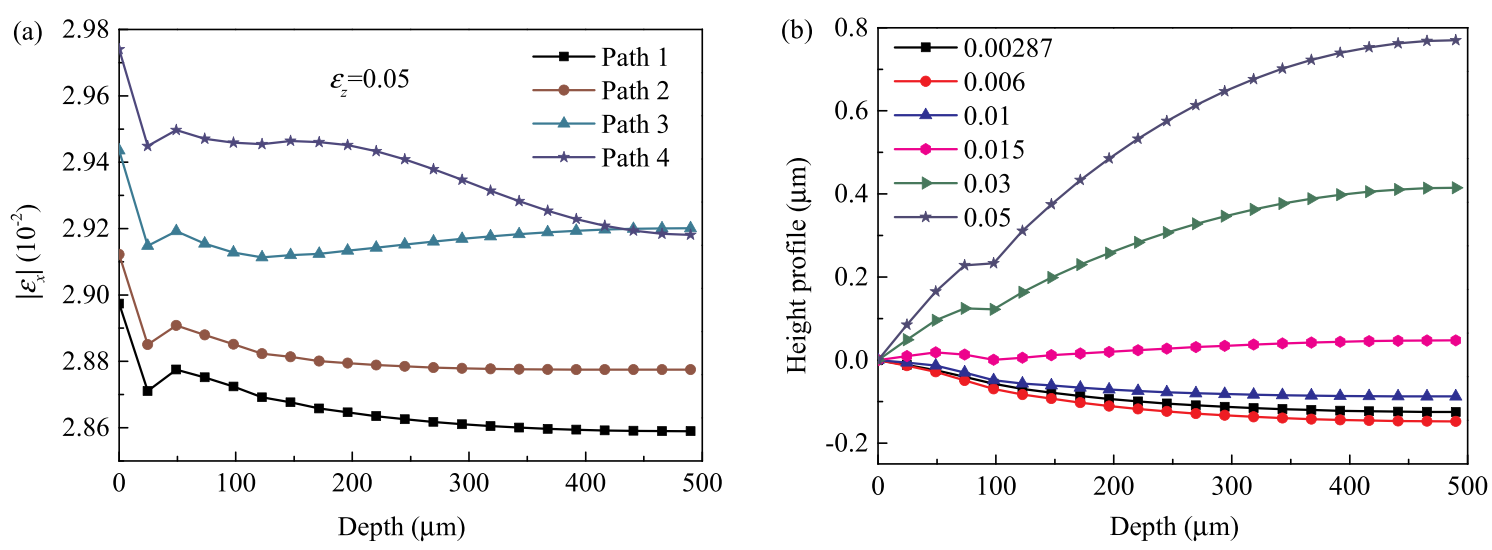

Fig. 9. (a) Distribution of lateral strain $\varepsilon_{x}$ along depth at a tensile strain of 0.05 . (b) Evolution of height profile in the lateral surface with tensile strain. 

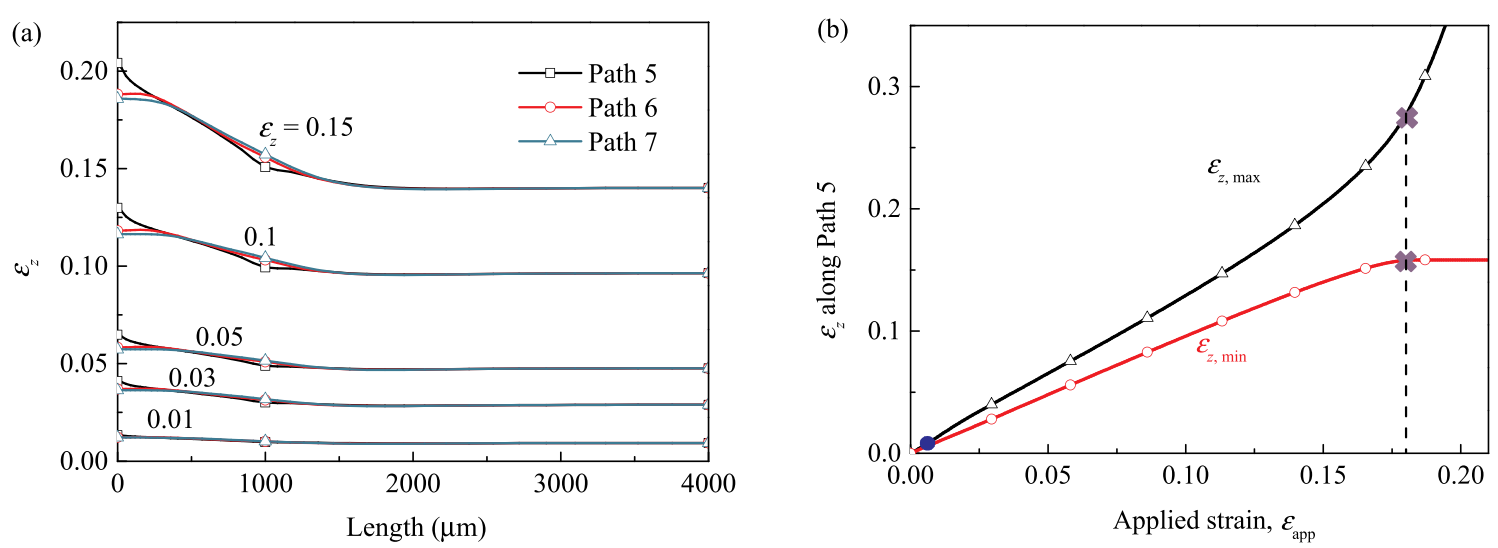

Fig. 10. (a) Evolutions of axial strain $\varepsilon_{z}$ along path 5 to path 7 with tensile strain. (b) Evolutions of the maximum and minimum axial strains $\varepsilon_{z \text {, max }}$ and $\varepsilon_{z, \min }$ with applied strain $\varepsilon_{\mathrm{z}}$.

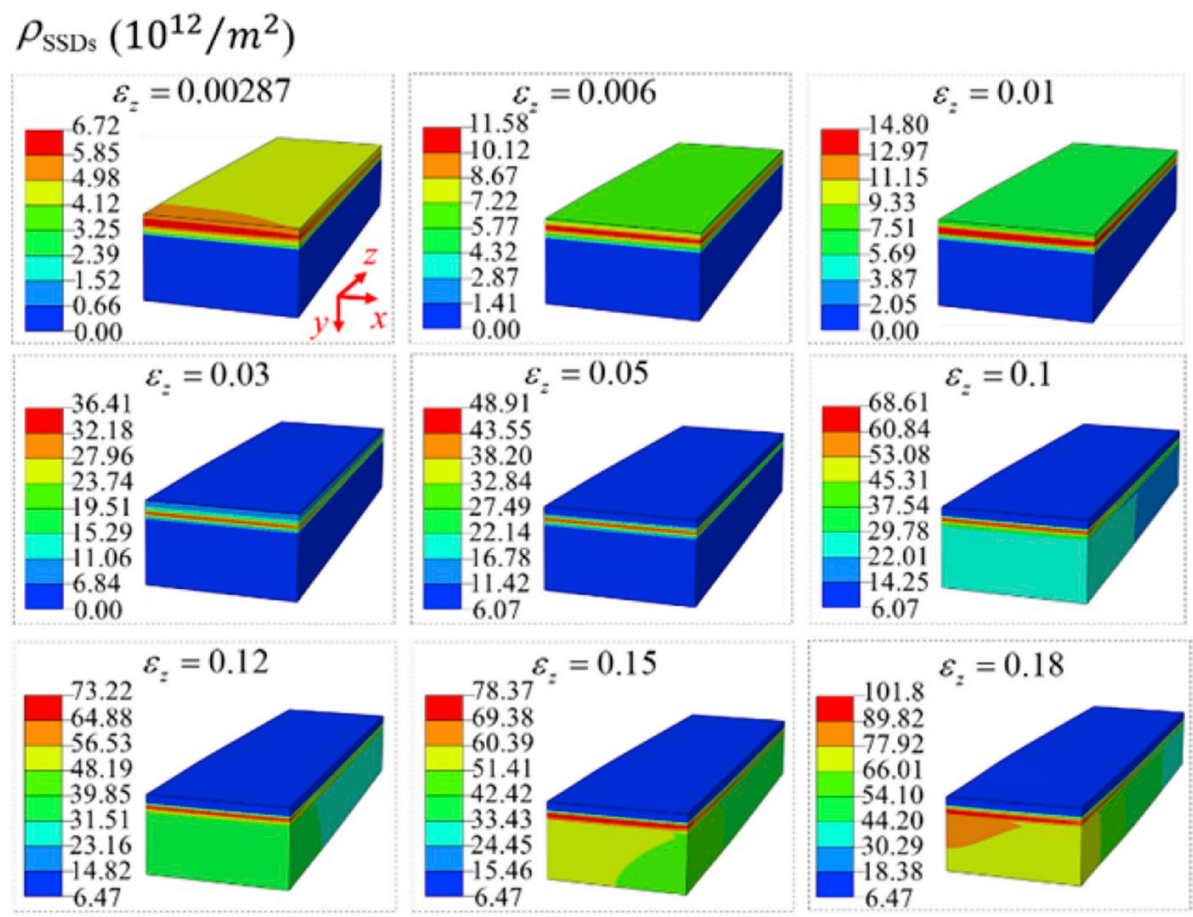

Fig. 11. Evolution of $\rho_{\text {SSDs }}$ with tensile strain.

distribution shown in Fig. 8(d), multiaxial stresses present in the GNG material and the highest lateral stress $\sigma_{x}$ occurs at the border demarcating the GNG layer and CG core. Thus, it is suspected that the multiaxial stresses help to promote dislocation multiplication and elevate the hardness at the border.

\subsubsection{GNDs}

The strain gradient at the sample level develops not only along the lateral $(x)$ direction but also along the loading ( $z$ ) direction. The non-uniform deformation at sample level gives rise to the construction of GNDs. Fig. 12(a) shows the distribution and evolution of $\rho_{\mathrm{GNDs}}^{\mathrm{sam}}$. It can be seen that the sample-level GNDs distribute mainly in GNG layer, and the maximum GNDs density appears at the border demarcating the GNG layer and the CG core. With the true strain increases from 0.00287 to 0.18 , the maximum GNDs density increases from $1.710^{11} / \mathrm{m}^{2}$ to $1.9610^{12} / \mathrm{m}^{2}$. Comparing with the density and distribution of SSDs, $\rho_{\mathrm{GNDs}}^{\text {sam }}$ can be neglected.

Fig. 12(b) illustrates the distribution and evolution of $\rho_{\mathrm{GNDs}}^{\text {gra }}$. Although the pileup dislocations in the GNG layer with smaller grain sizes are fewer than that in CG core, $\rho_{\mathrm{GNDs}}^{\text {gra }}$ in GNG region is higher since $\rho_{\mathrm{GNDs}}^{\text {gra }} \propto d^{2}$, as indicated by Malygin (2007). So $\rho_{\mathrm{GNDs}}^{\text {gra }}$ in the topmost surface layer with a grain size of $100 \mathrm{~nm}$ and with only one pileup dislocation inside grain is as high as $10^{14} / \mathrm{m}^{2}$ at a true strain 
(a) $\rho_{\text {GNDs }}^{\mathrm{sam}}\left(10^{12} / \mathrm{m}^{2}\right)$

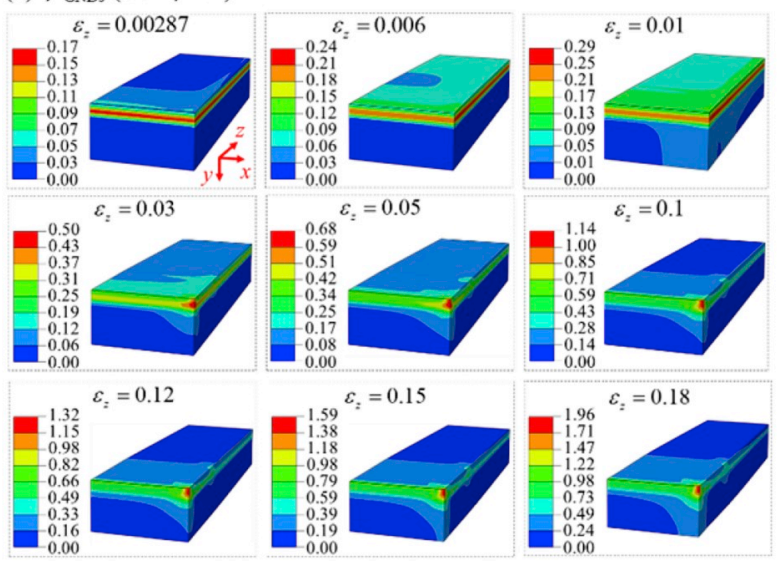

(b) $\rho_{\text {GNDs }}^{\text {gra }}\left(10^{12} / m^{2}\right)$

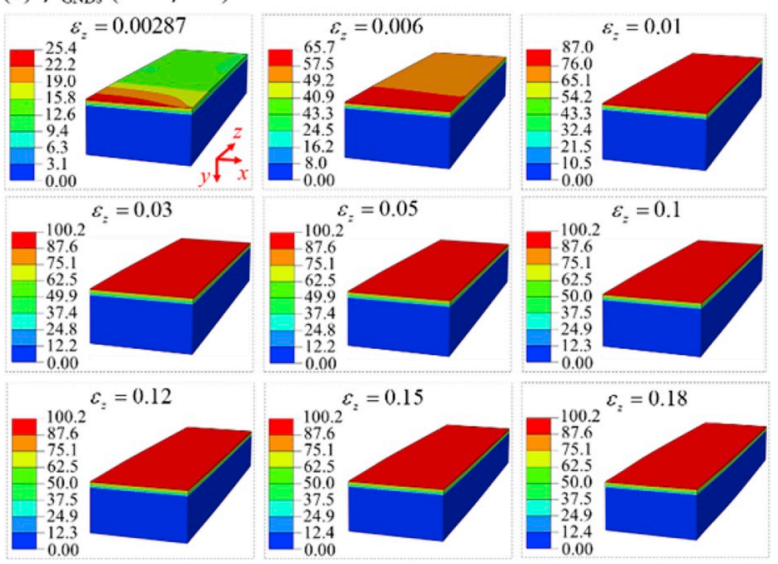

Fig. 12. (a) Distribution and evolution of $\rho_{\mathrm{GNDs}}^{\mathrm{sam}}$ and (b) $\rho_{\mathrm{GNDs}}^{\mathrm{gra}}$ with increasing tensile strain.

of 0.18 , which is two orders of magnitude higher than $\rho_{\mathrm{GNDs}}^{\text {sam }}$. Moreover, $\rho_{\mathrm{GNDs}}^{\text {gra }}$ saturates at an early deformation stage, i.e., the maximum value of $\rho_{\mathrm{GNDs}}^{\mathrm{gra}}$ remains as a constant after a tensile strain of 0.03 . It is because the pileup space for dislocations is limited in the surface grains, so the pileup dislocations saturate faster than that in the CG core.

\subsubsection{Back stress}

If back stress is not considered in the established constitutive model, the simulated flow stress in Fig. 7(a) is much lower than the experimental result. It's found that back stress increases progressively during the initial deformation stage. Thus, it influences not only the subsequent strain hardening behavior but also elevates the initial yield stress. To evaluate the effect of grain size gradient on back stress, the results of CG IF-steel with a grain size of $35 \mu \mathrm{m}$, both predicted by modeling and measured by experiments, are shown in Fig. 7(b). It is natural that the modeling prediction is also effective for CG material. The back stress of GNG material is about 50 MPa larger than the CG IF-steel, indicating that the GNG layer contributes to the extra strain hardening through back stress.

Fig. 13(a) and (b) show the evolutions of $\sigma_{\mathrm{b}}^{\text {sam }}$ and $\sigma_{\mathrm{b}}^{\text {gra }}$ with tensile strain. Fig. 13(a) indicates that $\sigma_{\mathrm{b}}^{\text {sam }}$ mainly distributes in the outer surface, and its maximum appears at the border demarcating the GNG layer and the CG core, where the largest gradient of $\rho_{\mathrm{GNDS}}^{\text {sam }}$ occurs. The maximum of $\sigma_{\mathrm{b}}^{\text {sam }}$ is only $0.28 \mathrm{MPa}$. In comparison, $\sigma_{\mathrm{b}}^{\text {gra }}$ can be as high as $271.5 \mathrm{MPa}$ in the surface region and $91.2 \mathrm{MPa}$ in the CG core at a tensile strain of 0.18, as shown in Fig. 13(b). Comparing Figs. 13(b) and 12(b), it's found that both the distribution and evolution of $\sigma_{\mathrm{b}}^{\text {gra }}$ are akin to that of $\rho_{\mathrm{GNDs}}^{\text {gra }}$, which is consistent with the constitutive descriptions that $\sigma_{\mathrm{b}}^{\text {gra }}$ and $\rho_{\mathrm{GNDs}}^{\text {gra }}$ are both controlled by the number of pileup dislocation $N$ in a similar way, as indicated by Eqs. (23) and (18).

\subsection{Strengthening mechanisms of the GNG IF-steel}

To further quantify the individual contribution of $\rho_{\mathrm{GNDs}}^{\mathrm{sam}}, \sigma_{\mathrm{b}}^{\mathrm{sam}}, \rho_{\mathrm{GNDs}}^{\mathrm{gra}}, \sigma_{\mathrm{b}}^{\mathrm{gra}}$ and $\rho_{\mathrm{SSDs}}$ to the overall stress-strain response of GNG IFsteel under uniaxial tension, five modeling cases are designed to separate their respective contribution, as shown in Table 2 . Where " $\sqrt{ }$ " means consider this factor and " " denotes eliminate its influence. Thus, case I indicates all mechanisms are included. Physically, $\sigma_{\mathrm{b}}^{\mathrm{sam}}$ and $\sigma_{\mathrm{b}}^{\text {gra }}$ are introduced by $\rho_{\mathrm{GNDs}}^{\mathrm{sam}}$ and $\rho_{\mathrm{GNDs}}^{\mathrm{gra}}$, respectively. Therefore, case II to case $\mathrm{V}$ are hypothetic cases to help to distinguish the individual contribution of GNDs density and the resulting back stress.

Fig. 14(a) shows the simulated stress-strain curves corresponding to the five cases presented in Table 2. It's found that the predictions of the case I, case II, and case III are almost the same. In comparison, the flow stress for case IV is a little lower than that for cases I, the main contribution of $\rho_{\mathrm{GNDs}}^{\text {gra }}$ comes from the accumulation of pileup GNDs in the surface region, as shown in Fig. 12(b). Furthermore, the stress-strain curve for case V is much lower than that of case I, demonstrating $\sigma_{\mathrm{b}}^{\text {gra }}$ has a significant contribution to the strengthening of GNG IF-steel.

Fig. 14(b) further quantifies the evolution of flow stress from $\rho_{\mathrm{GNDs}}^{\mathrm{sam}}, \sigma_{\mathrm{b}}^{\mathrm{sam}}, \rho_{\mathrm{GNDs}}^{\text {gra }}, \sigma_{\mathrm{b}}^{\text {gra }}$ with strain by analyzing the differences of the curves in Fig. 14(a). GBs strengthening according to the Hall-Petch relation and the strengthening from SSDs are also provided. It is found that the flow stresses originated from $\rho_{\mathrm{GNDs}}^{\text {sam }}$ and $\sigma_{\mathrm{b}}^{\text {sam }}$ are almost $0 \mathrm{MPa}$, demonstrating $\rho_{\mathrm{GNDs}}^{\text {sam }}$ and the resulting $\sigma_{\mathrm{b}}^{\text {sam }}$ have hardly any influence on the strain hardening of GNG IF-steel. The negligible effects of $\rho_{\mathrm{GNDs}}^{\text {sam }}$ and $\sigma_{\mathrm{b}}^{\text {sam }}$ on the overall strain hardening can also be inferred from their low values and limited distributions, as shown in Figs. 12(a) and 13(a). These findings agree with the strain gradient plasticity theory (Fleck et al., 1994; Gao et al., 1999; Huang et al., 2004), where the macroscopic strain gradient has hardly any influences on the strain hardening when the sample size is in millimeter-scale. The contribution of $\rho_{\mathrm{GNDs}}^{\text {gra }}$ increases at first and then saturates at a small strain about 0.02 with a maximum of $\sim 13 \mathrm{MPa}$. $\sigma_{\mathrm{b}}^{\text {gra }}$ increases gradually to $\sim 110 \mathrm{MPa}$ at a true strain of 0.18 , demonstrating a significant influence on the flow stress of GNG IF-steel. Furthermore, since the CG core possesses a strong strain 


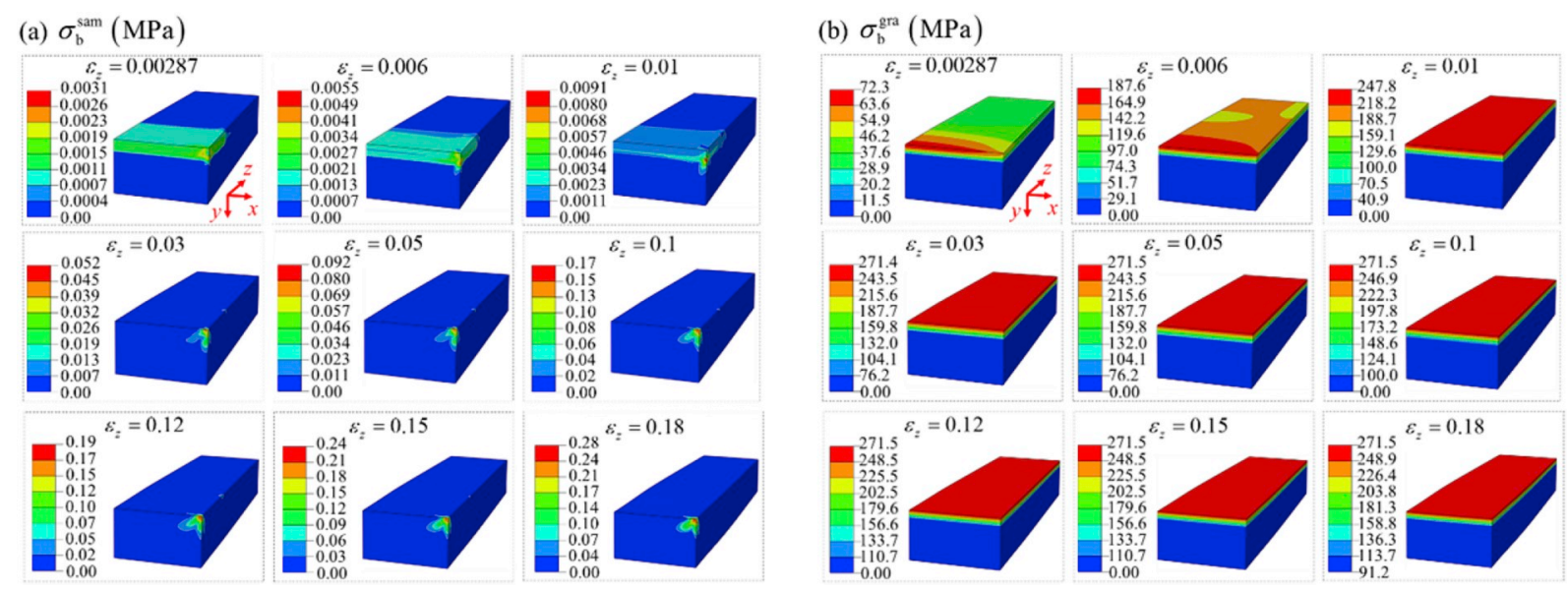

Fig. 13. (a) Evolution of $\sigma_{\mathrm{b}}^{\text {sam }}$ and (b) $\sigma_{\mathrm{b}}^{\text {gra }}$ with tensile strain.

Table 2

Modeling cases set to investigate the individual contribution of different strengthening factors.

\begin{tabular}{lllll}
\hline Case & $\rho_{\text {GNDs }}^{\text {sam }}$ & $\sigma_{\mathrm{b}}^{\text {sam }}$ & $\rho_{\text {GNDs }}^{\text {gra }}$ & $\sigma_{\mathrm{b}}^{\text {gra }}$ \\
\hline Case I & $\checkmark$ & $\checkmark$ & $\checkmark$ & $\checkmark$ \\
Case II & $\checkmark$ & $\checkmark$ & $\checkmark$ & $\checkmark$ \\
Case III & $\checkmark$ & $\checkmark$ & $\checkmark$ \\
Case IV & $\checkmark$ & $\checkmark$ & $\checkmark$ \\
Case V & $\checkmark$ & $\checkmark$ & $\checkmark$ & \\
\hline
\end{tabular}
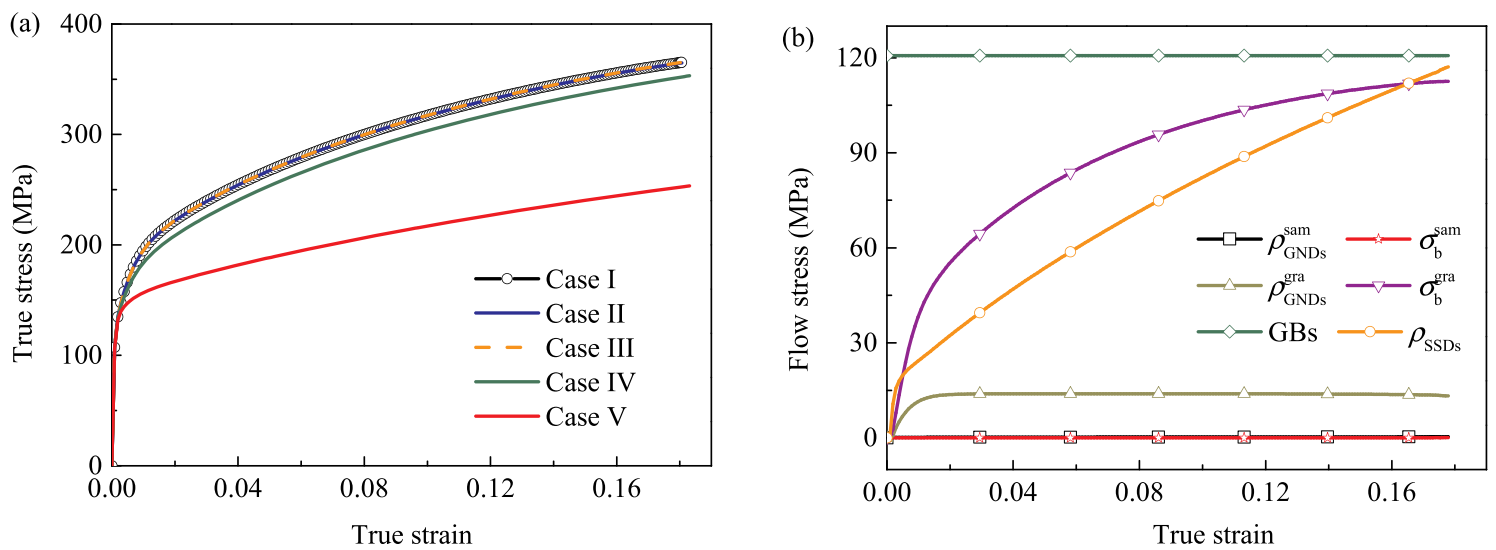

Fig. 14. (a) Simulated stress-strain curves corresponding to the five cases in Table 2. (b) Quantitative contributions of different factors to the flow stress of GNG IF-steel.

hardening capacity, strengthening comes from SSDs increases with the strain increasing throughout the entire deformation process to about $117 \mathrm{MPa}$ eventually. The contribution of GBs keeps a constant of about $120.7 \mathrm{MPa}$ because grain sizes are assumed to be constant during deformation.

The quantitative estimation of the effects of kinds of strengthening mechanisms on the stress-strain response of GNG IF-steel under tensile loading indicates that: the initial yielding is mainly controlled by the grain size effect characterized by the Hall-Petch relation, while the strain hardening is controlled by the SSDs, the pileup GNDs induced back stress $\sigma_{\mathrm{b}}^{\text {gra }}$ and the pileup GNDs themselves. Throughout the whole tensile deformation process of GNG material, it turns out that the strain gradient effect at the sample level (the GNDs accommodating macroscopic nonuniform deformation and the resulting $\sigma_{\mathrm{b}}^{\text {sam }}$ ) is negligible.

\section{Conclusions}

We develop a deformation-mechanism-based constitutive model considering the effects of grain size, GNDs and back stress on the 
strengthening and strain hardening of GNG materials and implement the developed model into a finite element framework to predict the tensile response of a GNG IF-steel sheet. Main conclusions of this work are drawn as follows:

(1) Multiaxial stresses develop due to mechanical incompatibilities of grains with different sizes and promote dislocation multiplication and strain hardening. The highest lateral stress appears at the border demarcating the GNG layer and CG core, where a peak $\rho_{\text {SSDs }}$ and sample-level GNDs $\rho_{\text {GNDs }}^{\text {sam }}$ occur by modeling prediction and a peak of hardness increment is also detected in experiments. The constraint between coarse-grained core on the GNG layer helps to suppress strain localization in the surface GNG layer from an arbitrary development along the depth direction, enabling the failure of GNG IF-steel to be delayed.

(2) At sample level, nonuniform deformation develops along both the depth and tensile directions, but the density of GNDs induced by the macroscopic strain gradient and the resulting back stress are too low to affect the strain hardening and strengthening of the GNG IF-steel.

(3) At grain level, strain gradient generates due to the slip discontinuities of adjacent grains, giving rise to pileup GNDs. The back stress resulting from pileup GNDs is demonstrated to contribute as high as 35\% to the strain hardening of the GNG IF-steel.

In the future, by manipulating the microstructure and sample size, the established model can be used to predict the relationship between microstructure and the strength-ductility of other kinds of GNG materials. The mechanical properties under complicated loading cases can also be analyzed.

\section{Declaration of competing interest}

We declare that we do not have any commercial or associative interest that represents a conflict of interest in connection with the work submitted.

\section{Acknowledgments}

This work was supported by the National Natural Science Foundation of China (Grant No. 11672251, 11872321) and the Opening Fund of State Key Laboratory of Nonlinear Mechanics.

\section{Appendix A. Supplementary data}

Supplementary data to this article can be found online at https://doi.org/10.1016/j.ijplas.2019.09.018.

\section{References}

ABAQUS, 2014. ABAQUS/Standard User's Manual Version 6.

Armstrong, R.W., 1970. The influence of polycrystalline grain size on mechanical properties. Adv. Mater. Res. 4, $101-146$.

Ashby, M.F., 1970. The deformation of plastically non-homogeneous materials. Philos. Mag. 21, 399-424.

Bayley, C.J., Brekelmans, W.A.M., Geers, M.G.D., 2006. A comparison of dislocation induced back stress formulations in strain gradient crystal plasticity. Int. J. Solids Struct. 43, 7268-7286.

Cheng, Z., Zhou, H., Lu, Q., Gao, H., Lu, L., 2018. Extra strengthening and work hardening in gradient nanotwinned metals. Science 362.

Chokshi, A.H., Rosen, A., Karch, J., Gleiter, H., 1989. On the validity of the hall-petch relationship in nanocrystalline materials. Scr. Metall. 23 , 1679-1683.

Espinosa, H.D., Panico, M., Berbenni, S., Schwarz, K.W., 2006. Discrete dislocation dynamics simulations to interpret plasticity size and surface effects in freestanding FCC thin films. Int. J. Plast. 22, 2091-2117.

Estrin, Y., 1998. Dislocation theory based constitutive modelling: foundations and applications. J. Mater. Process. Technol. 80, 33-39.

Estrin, Y., Mecking, H., 1984. A unified phenomenological description of work hardening and creep based on one-parameter models. Acta Metall. 32, 57-70.

Estrin, Y., Toth, L.S., Molinari, A., Brechet, Y., 1998. A dislocation-based model for all hardening stages in large strain deformation. Acta Mater. 46, 5509-5522.

Evers, L.P., Brekelmans, W.A.M., Geers, M.G.D., 2004. Non-local crystal plasticity model with intrinsic SSD and GND effects. J. Mech. Phys. Solids 52, $2379-2401$.

Evers, L.P., Brekelmans, W.A.M., Geers, M.G.D., 2004. Scale dependent crystal plasticity framework with dislocation density and grain boundary effects. Int. J. Solids Struct. 41, 5209-5230.

Fang, T.H., 2014. Fabrication, Mechanical Properties and Deformation Behavior of Gradient Nanograined (GNG) Structures. Ph.D. Thesis. University of Chinese Academy of Sciences, China.

Fang, T.H., Li, W.L., Tao, N.R., Lu, K., 2011. Revealing extraordinary intrinsic tensile plasticity in gradient nano-grained copper. Science 331, 1587-1590.

Fleck, N.A., Hutchinson, J.W., 1997. Strain gradient plasticity. Adv. Appl. Mech. 33, 295-361.

Fleck, N.A., Muller, G.M., Ashby, M.F., Hutchinson, J.W., 1994. Strain gradient plasticity: theory and experiment. Acta Metall. Mater. 42 , $475-487$.

Gao, H., Huang, Y., Nix, W.D., Hutchinson, J.W., 1999. Mechanism-based strain gradient plasticity- I. Theory. J. Mech. Phys. Solids 47, $1239-1263$.

Hall, E.O., 1951. The deformation and ageing of mild steel: III Discussion of results. Proc. Phys. Soc. Sect. B 64, 747-753.

Hill, R., 1950. The Mathematical Theory of Plasticity (E. Ullrich), 6, pp. 13-26.

Hirth, J.P., Lothe, J., 1982. Theory of Dislocations, seond ed. Wiley, New York.

Hu, X., Jin, S., Zhou, H., Yin, Z., Yang, J., Gong, Y., Zhu, Y., Sha, G., Zhu, X., 2017. Bauschinger effect and back stress in gradient Cu-Ge alloy. Metall. Mater. Trans. A 48, 3943-3950.

Huang, H.W., Wang, Z.B., Lu, J., Lu, K., 2015. Fatigue behaviors of AISI 316L stainless steel with a gradient nanostructured surface layer. Acta Mater. 87, 150-160. Huang, Y., Qu, S., Hwang, K.C., Li, M., Gao, H., 2004. A conventional theory of mechanism-based strain gradient plasticity. Int. J. Plast. 20, 753-782.

Jin, H., Zhou, J., Chen, Y., 2018. Grain size gradient and length scale effect on mechanical behaviors of surface nanocrystalline metals. Mater. Sci. Eng. A 725, $1-7$. Khan, A.S., Liu, J., 2016. A deformation mechanism based crystal plasticity model of ultrafine-grained/nanocrystalline FCC polycrystals. Int. J. Plast. 86, 56-69. Kim, H.S., Bush, M.B., 1999. The effects of grain size and porosity on the elastic modulus of nanocrystalline materials. Nanostructured Mater. 11, 361-367.

Kocks, U.F., 1976. Laws for work-hardening and low-temperature creep. Journal of Engineering Materials and Technology, Transactions of the ASME 98, 76-85. 
Kok, S., Beaudoin, A.J., Tortorelli, D.A., 2002. On the development of stage IV hardening using a model based on the mechanical threshold. Acta Mater. 50, $1653-1667$.

Kok, S., Beaudoin, A.J., Tortorelli, D.A., 2002. A polycrystal plasticity model based on the mechanical threshold. Int. J. Plast. 18, 715-741.

Kumar, K.S., Van Swygenhoven, H., Suresh, S., 2003. Mechanical behavior of nanocrystalline metals and alloys. Acta Mater. 51, 5743-5774.

Li, B.L., Godfrey, A., Meng, Q.C., Liu, Q., Hansen, N., 2004. Microstructural evolution of IF-steel during cold rolling. Acta Mater. 52, $1069-1081$.

Li, J., Chen, S., Wu, X., Soh, A., Lu, J., 2010. The main factor influencing the tensile properties of surface nano-crystallized graded materials. Mater. Sci. Eng. A 527, $7040-7044$.

Li, J., Soh, A., 2012. Enhanced ductility of surface nano-crystallized materials by modulating grain size gradient. Model. Simul. Mater. Sci. Eng. 20, 085002.

Li, J., Soh, A., 2012. Modeling of the plastic deformation of nanostructured materials with grain size gradient. Int. J. Plast. 39, 88-102.

Li, J., Weng, G., Chen, S., Wu, X., 2017. On strain hardening mechanism in gradient nanostructures. Int. J. Plast. 88, 89-107.

Lin, Y., Pan, J., Zhou, H.F., Gao, H.J., Li, Y., 2018. Mechanical properties and optimal grain size distribution profile of gradient grained nickel. Acta Mater. 153, 279-289.

Liu, X., Yuan, F., Zhu, Y., Wu, X., 2018. Extraordinary Bauschinger effect in gradient structured copper. Scr. Mater. 150, 57-60.

Long, J., Pan, Q., Tao, N., Dao, M., Suresh, S., Lu, L., 2019. Improved fatigue resistance of gradient nanograined Cu. Acta Mater. $166,56-66$.

Lu, K., 2014. Making strong nanomaterials ductile with gradients. Science 345, 1455-1456.

Lu, S., Zhang, B., Li, X., Zhao, J., Zaiser, M., Fan, H., Zhang, X., 2019. Grain boundary effect on nanoindentation: a multiscale discrete dislocation dynamics model. J. Mech. Phys. Solids 126, 117-135.

Lu, X., Zhang, X., Shi, M., Roters, F., Kang, G., Raabe, D., 2019. Dislocation mechanism based size-dependent crystal plasticity modeling and simulation of gradient nano-grained copper. Int. J. Plast. 113, 52-73.

Ma, E., Zhu, T., 2017. Towards strength-ductility synergy through the design of heterogeneous nanostructures in metals. Mater. Today $20,323-331$.

Mahato, J.K., De, P.S., Sarkar, A., Kundu, A., Chakraborti, P.C., 2016. Effect of deformation mode and grain size on Bauschinger behavior of annealed copper. Int. J. Fatigue 83, 42-52.

Malygin, G., 2007. Plasticity and strength of micro-and nanocrystalline materials. Phys. Solid State 49, 1013-1033.

Massart, T.J., Pardoen, T., 2010. Strain gradient plasticity analysis of the grain-size-dependent strength and ductility of polycrystals with evolving grain boundary confinement. Acta Mater. 58, 5768-5781.

Mecking, H., Kocks, U.F., 1981. Kinetics of flow and strain-hardening. Acta Metall. 29, 1865-1875.

Meyers, M.A., Mishra, A., Benson, D.J., 2006. Mechanical properties of nanocrystalline materials. Prog. Mater. Sci. 51, 427-556.

Moering, J., Ma, X., Malkin, J., Yang, M., Zhu, Y., Mathaudhu, S., 2016. Synergetic strengthening far beyond rule of mixtures in gradient structured aluminum rod. Scr. Mater. 122, 106-109.

Mughrabi, H., 1983. Dislocation wall and cell structures and long-range internal stresses in deformed metal crystals. Acta Metall. 31, 1367-1379.

Nye, J.F., 1953. Some geometrical relations in dislocated crystals. Acta Metall. 1, 153-162.

Ovid'ko, I.A., Valiev, R.Z., Zhu, Y.T., 2018. Review on superior strength and enhanced ductility of metallic nanomaterials. Prog. Mater. Sci. 94, 462-540.

Petch, N.J., 1953. The cleavage strength of polycrystals. Journal of the Iron and Steel Institute 174, 25-28.

Qu, S., 2004. A Conventional Theory of Mechanism-Based Strain Gradient Plasticity.

Roters, F., Raabe, D., Gottstein, G., 2000. Work hardening in heterogeneous alloys - a microstructural approach based on three internal state variables. Acta Mater. 48, $4181-4189$.

Schiotz, J., Jacobsen, K.W., 2003. A maximum in the strength of nanocrystalline copper. Science 301, $1357-1359$.

Shao, C.W., Zhang, P., Zhu, Y.K., Zhang, Z.J., Pang, J.C., Zhang, Z.F., 2017. Improvement of low-cycle fatigue resistance in TWIP steel by regulating the grain size and distribution. Acta Mater. 134, 128-142.

Shao, C., Zhang, P., Zhu, Y., Zhang, Z., Tian, Y., Zhang, Z., 2018. Simultaneous improvement of strength and plasticity: additional work-hardening from gradient microstructure. Acta Mater. 145, 413-428.

Sharma, P., Ganti, S., 2003. On the grain-size-dependent elastic modulus of nanocrystalline materials with and without grain-boundary sliding. J. Mater. Res. 18, 1823-1826.

Sinclair, C., Poole, W., Brechet, Y., 2006. A model for the grain size dependent work hardening of copper. Scr. Mater. 55, 739-742.

Stolken, J.S., Evans, A.G., 1998. A microbend test method for measuring the plasticity length scale. Acta Mater. 46, 5109-5115.

Taylor, G.I., 1934. The mechanism of plastic deformation of crystals. Part I. Theoretical. Proceedings of the Royal Society of London. Series A 145, 362-387.

Taylor, G.I., 1938. Plastic strain in metals. J. Inst. Met. 62, 307-324.

Valiev, R., Kozlov, E., Ivanov, Y.F., Lian, J., Nazarov, A., Baudelet, B., 1994. Deformation behaviour of ultra-fine-grained copper. Acta Metall. Mater. 42, $2467-2475$.

Van Swygenhoven, H., 2008. Footprints of plastic deformation in nanocrystalline metals. Mater. Sci. Eng. A 483, 33-39.

Wang, H., You, Z., Lu, L., 2018. Kinematic and isotropic strain hardening in copper with highly aligned nanoscale twins. Materials Research Letters 6, 333-338.

Wang, Y., Yang, G., Wang, W., Wang, X., Li, Q., Wei, Y., 2017. Optimal stress and deformation partition in gradient materials for better strength and tensile ductility: a numerical investigation. Sci. Rep. 7, 10954.

Wei, Y., Li, Y., Zhu, L., Liu, Y., Lei, X., Wang, G., Wu, Y., Mi, Z., Liu, J., Wang, H., 2014. Evading the strength-ductility trade-off dilemma in steel through gradient hierarchical nanotwins. Nat. Commun. 5, 1-8.

Wu, X., Jiang, P., Chen, L., Yuan, F., Zhu, Y.T., 2014. Extraordinary strain hardening by gradient structure. Proc. Natl. Acad. Sci. U.S.A. $111,7197$.

Wu, X., Zhu, Y., 2017. Heterogeneous materials: a new class of materials with unprecedented mechanical properties. Materials Research Letters 5, 527-532.

Wu, X.L., Jiang, P., Chen, L., Zhang, J.F., Yuan, F.P., Zhu, Y.T., 2014. Synergetic strengthening by gradient structure. Materials Research Letters 2, 185-191.

Yang, M., Pan, Y., Yuan, F., Zhu, Y., Wu, X., 2016. Back stress strengthening and strain hardening in gradient structure. Materials Research Letters 4, 145-151.

Yang, X., Ma, X., Moering, J., Zhou, H., Wang, W., Gong, Y., Tao, J., Zhu, Y., Zhu, X., 2015. Influence of gradient structure volume fraction on the mechanical properties of pure copper. Mater. Sci. Eng. A 645, 280-285.

Yin, Z., Yang, X., Ma, X., Moering, J., Yang, J., Gong, Y., Zhu, Y., Zhu, X., 2016. Strength and ductility of gradient structured copper obtained by surface mechanical attrition treatment. Mater. Des. 105, 89-95.

Yuan, F., Yan, D., Sun, J., Zhou, L., Zhu, Y., Wu, X., 2019. Ductility by shear band delocalization in the nano-layer of gradient structure. Materials Research Letters 7, $12-17$.

Zeng, Z., Li, X., Xu, D., Lu, L., Gao, H., Zhu, T., 2015. Gradient plasticity in gradient nano-grained metals. Extreme Mechanics Letters 213-219.

Zhao, J., Kan, Q., Zhou, L., Kang, G., Fan, H., Zhang, X., 2019. Deformation mechanisms based constitutive modelling and strength-ductility mapping of gradient nano-grained materials. Mater. Sci. Eng. A 742, 400-408.

Zhu, L., Lu, J., 2012. Modelling the plastic deformation of nanostructured metals with bimodal grain size distribution. Int. J. Plast. 30, 166-184. 